\title{
A spacetime characterization of the Kerr-NUT-(A)de Sitter and related metrics
}

\author{
Marc Mars ${ }^{1}$ and José M. M. Senovilla ${ }^{2}$ \\ ${ }^{1}$ Instituto de Física Fundamental y Matemáticas, Universidad de Salamanca \\ Plaza de la Merced s/n, 37008 Salamanca, Spain \\ marc@usal.es \\ ${ }^{2}$ Física Teórica, Universidad del País Vasco, \\ Apartado 644, 48080 Bilbao, Spain \\ josemm.senovilla@ehu.es
}

\begin{abstract}
A characterization of the Kerr-NUT-(A)de Sitter metric among four dimensional $\Lambda$-vacuum spacetimes admitting a Killing vector $\xi$ is obtained in terms of the proportionality of the self-dual Weyl tensor and a natural self-dual double two-form constructed from the Killing vector. This result recovers and extends a previous characterization of the Kerr and Kerr-NUT metrics [30]. The method of proof is based on (i) the presence of a second Killing vector field which is built in terms of geometric information arising from the Killing vector $\xi$ exclusively, and (ii) the existence of an interesting underlying geometric structure involving a Riemannian submersion of a conformally related metric, both of which may be of independent interest. Other related metrics can also be similarly characterized, in particular the $\Lambda<0$ "black branes" recently used in AdS/CFT correspondence to describe via holography the physics of Quark-Gluon plasma.
\end{abstract}

\section{Introduction}

The Kerr-NUT-(A)de Sitter spacetimes is a class of solutions of the Einstein field equations with cosmological constant $\Lambda$ (of any value) which includes the class of Kerr and Kerr-NUT spacetimes. These spacetimes are widely believed to play an important role as stationary endpoints of self-gravitating collapsing systems. Although we are very far from any rigorous result in this direction, substantial work has been made on this problem at the linear level, specially in the case of vanishing cosmological constant where there is a large body of literature concerning boundedness and decay properties of linear waves propagating in 
a Kerr background (see [8] and references therein). For the case of positive cosmological constant, it has been shown that linear waves in the Kerr-de Sitter background decay exponentially in time outside and including the event horizon (see [11] and references therein). In the case of negative cosmological constant, Holzegel has proven boundedness [20] of the Klein-Gordon equation in Kerr-Anti de Sitter backgrounds and Holzegel and Smulevici [21] have shown logarithmic decay of the solutions. This very slow decay suggests non-linear instability of these spacetimes, and this behaviour has been conjectured for all asymptotically Anti-de Sitter spacetimes in [21] (see, however, [10] for heuristic arguments in favor of non-linear stability). All these considerations show the physical relevance of the class of Kerr-NUT-(A)de Sitter spacetimes, which makes it of interest to try and find a geometric characterization for them.

In the case of the Kerr metric (and also Kerr-NUT metric), a spacetime characterization within the class of vacuum spacetimes admitting a Killing vector $\xi$ was found in [30] motivated by previous results [43] in the quotient manifold of the Killing field in the region where the Killing is timelike. This characterization was local in nature (see [32] for a discussion of this point) and involved the proportionality of the self-dual Weyl tensor and a tensor with the same algebraic properties constructed out of the Killing vector $\xi$. The characterization assumed implicitly a property never spelled out, namely, that the Killing vector was nowhere orthogonal to the 2-plane spanned by the two principal directions of the Weyl tensor, see [35] where this omission was corrected and the accurate result explained in detail. Of course, the omitted assumption is automatically satisfied if the Killing vector is timelike somewhere. Nevertheless, the characterization of Kerr in [30, 31] has played an interesting role in trying to extend the uniqueness of stationary black holes from the analytic setting to the general case [24], [1]. It has also been used to characterize initial data for the Kerr metric in [15] and, more recently, to define a quality factor measuring the deviation of a given metric admitting a Killing vector with respect to the Kerr metric [14]. A characterization of the Kerr-Newman spacetime in the same spirit has been obtained by Wong [46].

Given the natural relation between the Kerr (and Kerr-NUT) metrics and the KerrNUT-(A)de Sitter metrics, it is most natural to ask whether the characterization above can be extended to the larger class of spacetimes. This is the problem we address in this paper. The derivations in [30] and [46] used extensively the Newman-Penrose formalism to find local properties of the spacetimes under consideration. Here we follow a completely different approach which allows us to dispense of the use of the Newman-Penrose formalism altogether. This has the advantage that the underlying geometric structures become much more clearly exposed. As we will see, two such structures will play a fundamental role. The first one involves a Riemannian submersion (of codimension two) involving a conformally related metric, where the conformal factor is determined in terms of objects constructed from the Killing vector $\xi$. The second one is the presence of a second Killing vector field in the spacetime. This Killing field is again constructed solely in terms of geometric information arising from the Killing vector $\xi$. The explicit form of the second Killing field may have potential interest in other areas of research, such as, for instance, in extending the Hawking rigidity theorem for stationary black holes from the analytic to the smooth setting (see [2], 
[3], 25] for interesting developments in this direction). We emphasize that the cosmological constant $\Lambda$ is not assumed to be non-zero anywhere in the paper, so all our results apply to the vacuum case as well. As already mentioned, the proof presented here is very different to the proofs in [30] and 31] which were based in a tetrad approach. The method in [30] was based on constructing a tetrad of commuting vector fields, two of which were Killing vector fields of the spacetime. There is no simple parallelism between this proof and the one presented here. The method in [31], although still based on tetrads, already has some of the ingredients we exploit here. More specifically a two-dimensional distribution and its corresponding quotient space also plays a fundamental role in integrating the field equations in 31] (cf. Proposition 2 in this reference). However, no isometric submersion is identified in that paper. The geometrically much more transparent proof presented here is not only able to deal with Einstein spaces but also reveals interesting geometric structure that helps understanding the vacuum characterization as well.

Given that the characterization condition we impose is fully local in nature, it is clear that no global restrictions on the spacetime can be deduced from it. Our aim is therefore to prove a local isometry to the Kerr-NUT-(A)de Sitter spacetime in the following precise sense.

Definition 1. Given real constants $\{\Lambda, m, a, l\}$ for which the function $\Delta_{\theta}:=1+\frac{\Lambda}{3} a \cos \theta(4 l+$ $a \cos \theta)$ is everywhere positive, a spacetime $(\mathcal{M}, g)$ is locally isometric to the Kerr-NUT(A) de Sitter spacetime with parameters $\{\Lambda, m, a, l\}$ if for any point $p \in \mathcal{M}$ where no Killing vector of $(\mathcal{M}, g)$ vanishes there is a neighbourhood $U_{p}$ of $p$ and coordinates $\{u, r, \theta, \phi\}$ on $U_{p}$ such that $g$ takes the local form

$$
\begin{aligned}
g= & -\frac{\Delta-a^{2} \sin ^{2} \theta \Delta_{\theta}}{\rho^{2}}\left(d u-\left(a \sin ^{2} \theta+4 l \sin ^{2}(\theta / 2)\right) d \phi\right)^{2}+2\left(d r-a \sin ^{2} \theta \Delta_{\theta} d \phi\right) \times \\
& \left(d u-\left(a \sin ^{2} \theta+4 l \sin ^{2}(\theta / 2)\right) d \phi\right)+\rho^{2}\left(\frac{d \theta^{2}}{\Delta_{\theta}}+\Delta_{\theta} \sin ^{2} \theta d \phi^{2}\right)
\end{aligned}
$$

where

$\rho^{2}:=r^{2}+(l+a \cos \theta)^{2}, \quad \Delta:=a^{2}-l^{2}-2 m r+r^{2}-\frac{\Lambda}{3}\left(3 l^{2}\left(a^{2}-l^{2}\right)+\left(a^{2}+6 l^{2}\right) r^{2}+r^{4}\right)$.

Note that, at points where $d r$ is non-null, the metric (11) can be transformed into the more usual form (see e.g. [17]):

$$
\begin{aligned}
g= & -\frac{\Delta}{\rho^{2}}\left(d t-\left(a \sin ^{2} \theta+4 l \sin ^{2}(\theta / 2)\right) d \varphi\right)^{2}+\frac{\Delta_{\theta} \sin ^{2} \theta}{\rho^{2}}\left(a d t-\left(r^{2}+(a+l)^{2}\right) d \varphi\right)^{2}+ \\
& +\frac{\rho^{2}}{\Delta} d r^{2}+\frac{\rho^{2}}{\Delta_{\theta}} d \theta^{2}
\end{aligned}
$$

by the coordinate transformation

$$
d t=d u-\frac{r^{2}+(a+l)^{2}}{\Delta} d r, \quad d \varphi=d \phi-\frac{a}{\Delta} d r .
$$

A suitable combination of the main results in this paper can be stated as the following theorem (see below for definitions) 
Theorem 1. Let $(\mathcal{M}, g)$ be a $\Lambda$-vacuum spacetime admitting a Killing vector $\xi$ with selfdual two-form $\mathcal{F}_{\alpha \beta}$. Assume there exists $Q \in C^{\infty}(\mathcal{M}, \mathbb{C})$ such that

$$
\mathcal{C}_{\alpha \beta \mu \nu}=Q\left(\mathcal{F}_{\alpha \beta} \mathcal{F}_{\mu \nu}-\frac{1}{3} \mathcal{F}^{2} \mathcal{I}_{\alpha \beta \mu \nu}\right),
$$

where $\mathcal{C}_{\alpha \beta \mu \nu}$ is the self-dual Weyl tensor of $g$ and $\mathcal{F}^{2} \stackrel{\text { def }}{=} \mathcal{F}_{\alpha \beta} \mathcal{F}^{\alpha \beta}$ and assume that $\exists p, p^{\prime}, p^{\prime \prime} \in$ $\mathcal{M}$ such that $\left.\left(Q \mathcal{F}^{2}\right)\right|_{p} \neq 0,\left.\left(Q \mathcal{F}^{2}-4 \Lambda\right)\right|_{p^{\prime}} \neq 0$, and $\left.\xi\right|_{p^{\prime \prime}}$ is not orthogonal to the 2-plane generated by the two real null eigenvectors of $\left.\mathcal{F}_{\alpha \beta}\right|_{p^{\prime \prime}}$. Then $\mathcal{F}^{2} \neq 0$ and $Q \mathcal{F}^{2}-4 \Lambda \neq 0$ everywhere and there exist constants $b_{1}, b_{2}, c, k \in \mathbb{R}$ such that

$$
\begin{array}{r}
36 Q\left(\mathcal{F}^{2}\right)^{\frac{5}{2}}+\left(b_{2}-i b_{1}\right)\left(Q \mathcal{F}^{2}-4 \Lambda\right)^{3}=0 \\
g(\xi, \xi)+\operatorname{Re}\left(\frac{6 \mathcal{F}^{2}\left(Q \mathcal{F}^{2}+2 \Lambda\right)}{\left(Q \mathcal{F}^{2}-4 \Lambda\right)^{2}}\right)+c=0 \\
-k+\left|\frac{36 \mathcal{F}^{2}}{\left(Q \mathcal{F}^{2}-4 \Lambda\right)^{2}}\right| \nabla_{\alpha} Z \nabla^{\alpha} Z-b_{2} Z+c Z^{2}+\frac{\Lambda}{3} Z^{4}=0
\end{array}
$$

where $Z=\operatorname{Im}\left(\frac{6 i \sqrt{\mathcal{F}^{2}}}{Q \mathcal{F}^{2}-4 \Lambda}\right)$. If these constants are such that the polynomial $V(\zeta):=k+b_{2} \zeta-$ $c \zeta^{2}-\frac{\Lambda}{3} \zeta^{4}$ can be factored as

$$
V(\zeta)=\hat{V}(\zeta)\left(\zeta-\zeta_{0}\right)\left(\zeta_{1}-\zeta\right)
$$

with $\zeta_{0} \leq \zeta_{1}$ and $\hat{V}(\zeta)>0$ on $\left[\zeta_{0}, \zeta_{1}\right]$ and $Z: \mathcal{M} \rightarrow\left[\zeta_{0}, \zeta_{1}\right]$ then $(\mathcal{M}, g)$ is locally isometric to the Kerr-NUT-(A)dS with parameters $\{\Lambda, m, a, l\}$ where

$$
m=\frac{b_{1}}{2 v_{0} \sqrt{v_{0}}}, \quad a=\frac{\zeta_{1}-\zeta_{0}}{2 \sqrt{v_{0}}}, \quad l=\frac{\zeta_{1}+\zeta_{0}}{2 \sqrt{v_{0}}}
$$

and $v_{0}:=\hat{V}\left(\frac{\zeta_{0}+\zeta_{1}}{2}\right)$.

In fact we obtain in Theorem 4 a full characterization of $\Lambda$-vacuum spacetimes for which (2) holds and for which $\mathcal{F}^{2}$ is not identically zero.

Other local characterization results for Kerr-NUT-(A)de Sitter spacetimes have been obtained based on the separability of the Hamilton-Jacobi equation. As it is well-known from the Kerr case, the underlying reason for this separability is the presence of hidden symmetries in the form of Killing tensors or, more generally, in the form of conformal KillingYano tensors. The presence of such symmetries of the Kerr-NUT-(A)de Sitter spacetime (in fact, on the corresponding class of metrics in arbitrary dimension, as given in [6]) was found in [29] (see also [7] for the particular case when all rotation parameters except one vanish). Results concerning the relationship between the Kerr-NUT-(A)de Sitter spacetimes and $\Lambda$-vacuum spacetimes admitting a closed conformal Killing-Yano tensor have been obtained in 23] (in arbitrary dimension) based on previous work in 22] and 28]. The results in these papers and the characterization of Kerr-NUT-(A)de Sitter presented here are a priori completely different. It is an interesting open problem whether there is any 
way of connecting both types of results. Besides its intrinsic interest, this would open up the possibility of extending the characterization results in this paper (which are restricted to four spacetime dimensions) to the higher dimensional case. In this respect, it is worth mentioning that a generalization [31] of the spacetime characterization of the Kerr spacetime in [30] has been used [4] (see also [12] for related statements) to obtain an alternative characterization of this metric involving Killing spinors (and hence hidden symmetries).

A final remark is in order. The hypotheses in our characterization theorems restrict the spacetime to be of Petrov type D on a non-empty open subset. However, our hypotheses allow a priori that the Weyl tensor degenerates to type $\mathrm{N}$ or type $\mathrm{O}$ elsewhere in the spacetime. In the field of exact solutions, $\Lambda$-vacuum spacetimes of Petrov type $\mathrm{D}$ have been thoroughly studied, and the most general class of such metrics has been found to be the so-called Plebański-Demiański metric and its limiting cases (this was analyzed by Deveber at al [9] and García [13], see also [18]). So, one might think that a convenient strategy to prove the characterization results in this paper would be to identify (within this general class) those metrics satisfying the proportionality condition between the self-dual Weyl tensor and the self-dual double two-form of the Killing vector. However, there are two main reasons why this is not so. First and foremost, in the field of exact solutions the aim is to find explicit Lorentzian metrics solving the Einstein field equations. Often the equations split into several cases depending on whether certain quantities vanish or not. The method typically proceeds by assuming that either the quantity is not zero or it vanishes on an open set. This is fine in this field of research, since the regions left out by the analysis are of empty interior. However, it is not quite sufficient for our purposes, where we want to identify the metric locally around any point in the given spacetime (or at least keeping full control of the points that are left out). Studying the transition between a priori disjoint regions, which may or may not belong to the same spacetime, is a difficult task in general. This makes the use of the exact solution results of little use in the present context. The second reason is related to the previous one. As already mentioned, main hypotheses do not fix a priori the Weyl tensor to be of Petrov type D everywhere in the spacetime so it becomes necessary to study what happens at the boundary (if any) of open set where the Petrov type is $D$. For this, a semiglobal analysis is required, which again precludes (or makes it difficult) the use of fully local results, as those in the field of exact solutions. All these considerations were also present in the case of vanishing $\Lambda$ studied in [30].

The plan of the paper is as follows. In Section 2 we review a number of known results for spacetimes admitting a Killing vector, especially those concerning the so-called self-dual Killing form and the Ernst one-form constructed from it. We first present results valid for general spacetimes and then we concentrate, in Subsection 2.1, on $\Lambda$-vacuum spacetimes. Algebraic and differential consequences of the proportionality between the self-dual Weyl tensor and the self-dual double Killing two-form are obtained in Subsection 2.2 (further results on the self-dual Killing form necessary for the paper are included in an Appendix). In Section 3 we show that the Ernst one-form is globally exact, and construct explicitly a second Killing vector in terms of the geometry of $\xi$. The two Killing vectors span a two-dimensional Killing algebra except in special cases, which are analyzed in detail in Subsection 3.1, as they require a separate treatment throughout the paper. In Section 4 
the field equations are solved by exploiting the local Riemannian submersion in a conformally related spacetime mentioned above. Our main results here are Theorems 2 and 3 where local forms of the metric are obtained around any point in the spacetime, except fixed points of the Killing vector $\xi$. Section 5 is devoted to showing first that the Petrov type must remain constant throughout the spacetime and understanding the relationship between the special cases and the generic cases. For this, the Killing algebra derived in Sect. 3 plays a fundamental role. Our last Section [6] is devoted to identifying the metrics obtained in the previous sections, and hence writing down our characterization results. First of all we summarize in Theorem 4 the main results of the previous sections in a selfcontained form, so as to avoid the need to refer to previous definitions in the reminder of the paper. Based on this result we obtain characterizations of the Plebański, uncharged Bertotti-Robinson and Nariai metrics in Theorem 5. Finally, the Kerr-NUT-(A)de Sitter metric is fully characterized in Theorem 6, and we also discuss similar characterizations of other related interesting metrics, such as the "black branes" (also called "planar" black holes) found in [27] and recently discussed in connection with the AdS/CFT holographic description of the Quark-Gluon plasma [36, 37, 38].

\section{Preliminaries}

Throughout this paper, a spacetime $(\mathcal{M}, q)$ is a smooth, orientable, four-dimensional, connected manifold endowed with a smooth 1 metric $g$ of Lorentzian signature. We assume further that the spacetime is time orientable and time oriented. The Levi-Civita covariant derivative of $g$ is denoted by $\nabla$ and the volume form by $\eta_{\alpha \beta \gamma \delta}$.

Our basic assumption is that the spacetime possesses a Killing vector field $\xi$, whose norm is denoted by $N \stackrel{\text { def }}{=}-\xi^{\mu} \xi_{\mu}$. The 2 -form $F_{\mu \nu} \stackrel{\text { def }}{=} \nabla_{\mu} \xi_{\nu}$ defines the complex self-dual Killing 2-form:

$$
\mathcal{F}_{\alpha \beta} \stackrel{\text { def }}{=} F_{\alpha \beta}+i F_{\alpha \beta}^{\star}, \quad \mathcal{F}_{\alpha \beta}^{\star}=-i \mathcal{F}_{\alpha \beta},
$$

where $\star$ is the Hodge dual operator. Well known identities for $\mathcal{F}$ (see e.g. [26]) are

$$
\mathcal{F}_{\mu \rho} \mathcal{F}_{\nu}{ }^{\rho}=\frac{1}{4} \mathcal{F}^{2} g_{\mu \nu}, \quad \mathcal{F}^{2} \stackrel{\text { def }}{=} \mathcal{F}_{\alpha \beta} \mathcal{F}^{\alpha \beta}, \quad \mathcal{F}_{\mu \rho} \overline{\mathcal{F}}^{\mu \rho}=0
$$

where overbars denote complex conjugation. The Ernst one-form is defined by

$$
\chi_{\beta} \stackrel{\text { def }}{=} 2 \xi^{\alpha} \mathcal{F}_{\alpha \beta}, \quad \xi^{\beta} \chi_{\beta}=0 \quad \chi_{\alpha} \chi^{\alpha}=-N \mathcal{F}^{2}
$$

the last relation being a consequence of (4) by contraction with $\xi$. Actually, by the same route one gets from (4)

$$
\chi^{\rho} \mathcal{F}_{\rho \mu}=-\frac{1}{2} \mathcal{F}^{2} \xi_{\mu}, \quad \mathcal{F}_{\mu \nu} \chi^{\mu} \bar{\chi}^{\nu}=0 .
$$

The real part of the Ernst one-form satisfies

$$
\chi_{\mu}+\bar{\chi}_{\mu}=4 \xi^{\rho} F_{\rho \mu}=-4 \xi^{\rho} \nabla_{\mu} \xi_{\rho}=2 \nabla_{\mu} N
$$

\footnotetext{
${ }^{1}$ Smoothness is assumed only for notational simplicity. All the results hold if the metric is merely $C^{3}$.
} 
so that we can always write

$$
\chi_{\mu}=\nabla_{\mu} N+i \omega_{\mu}
$$

for some real one-form $\omega$ called the twist of $\xi$. We also introduce the following real one-form

$$
\eta_{\mu} \stackrel{\text { def }}{=} \bar{\chi}^{\rho} \mathcal{F}_{\rho \mu}=\chi^{\rho} \overline{\mathcal{F}}_{\rho \mu}, \quad \bar{\eta}_{\mu}=\eta_{\mu}
$$

which satisfies

$$
\eta^{\rho} \mathcal{F}_{\rho \mu}=-\frac{1}{4} \mathcal{F}^{2} \bar{\chi}_{\mu}, \quad \eta_{\mu} \chi^{\mu}=0, \quad \eta_{\mu} \xi^{\mu}=-\frac{1}{2} \chi^{\rho} \bar{\chi}_{\rho}, \quad \eta_{\mu} \eta^{\mu}=-\frac{N}{4} \mathcal{F}^{2} \overline{\mathcal{F}}^{2} .
$$

This seems to be a better choice than the more "natural" real one-form $i \eta_{\alpha \beta \mu \nu} \bar{\chi}^{\beta} \xi^{\mu} \chi^{\nu}$, and in any case one has the following relations

$$
\boldsymbol{\xi} \wedge \boldsymbol{\eta}=\frac{i}{2} \star(\chi \wedge \overline{\boldsymbol{\chi}}) \Longrightarrow i \eta_{\alpha \beta \mu \nu} \bar{\chi}^{\beta} \xi^{\mu} \chi^{\nu}=2 N \eta_{\alpha}-\left(\chi^{\rho} \bar{\chi}_{\rho}\right) \xi_{\alpha}
$$

where we use bold symbols to refer to $p$-forms (in particular covectors) when no abstract indices are used. We will also use the following symmetric tensor

$$
t_{\mu \nu} \stackrel{\text { def }}{=} \frac{1}{2} \mathcal{F}_{\mu \rho} \overline{\mathcal{F}}_{\nu}^{\rho}
$$

which is nothing but the "energy-momentum tensor" of the 2 -form $F_{\mu \nu}$. Well known properties of this tensor are (cf. (44))

$$
t_{\mu \nu}=t_{\nu \mu}, \quad t_{\rho}^{\rho}=0, \quad t_{\mu \rho} t_{\nu}{ }^{\rho}=\frac{1}{4} t_{\rho \sigma} t^{\rho \sigma} g_{\mu \nu}, \quad t_{\rho \sigma} t^{\rho \sigma}=\frac{1}{16} \mathcal{F}^{2} \overline{\mathcal{F}}^{2} .
$$

Note however that it is not divergence-free in general, as $\mathcal{F}$ does not necessarily satisfy the Maxwell equations in vacuum.

As $\xi$ is a Killing vector we obviously have

$$
£_{\xi} \mathcal{F}_{\mu \nu}=0, \quad £_{\xi} t_{\mu \nu}=0, \quad \xi\left(\mathcal{F}^{2}\right)=0, \quad \xi(N)=0, \quad[\xi, \chi]=0, \quad[\xi, \eta]=0 .
$$

Further properties of all these objects are collected in an Appendix.

A complex self-dual 2-form $\mathcal{F}_{\mu \nu}$ is said to be regular at $p \in \mathcal{M}$ if $\left.\mathcal{F}^{2}\right|_{p} \neq 0$. If $\left.\mathcal{F}^{2}\right|_{p}=0$ then $\mathcal{F}_{\mu \nu}$ is called singular. In the regular case, there exist two different real (and necessarily null) eigenvectors $k_{ \pm}$of $\mathcal{F}_{\mu \nu}$ at $p$ with opposite eigenvalues $\pm R$

$$
k_{ \pm}^{\nu} \mathcal{F}_{\mu \nu}= \pm R k_{ \pm \mu}, \quad g\left(k_{ \pm}, k_{ \pm}\right)=0
$$

while there exists only one, $k_{+}=k_{-}$, with zero eigenvalue, in the singular case. Then $k_{ \pm}$ are also eigenvectors of $t_{\mu \nu}$ and we have

$$
k_{ \pm}^{\mu} t_{\mu \nu}=-\frac{1}{2} R \bar{R} k_{\nu}^{ \pm}, \quad \mathcal{F}^{2}=-4 R^{2}
$$


as well as

$$
g\left(k_{ \pm}, \chi\right)= \pm 2 R g\left(\xi, k_{ \pm}\right), \quad g\left(k_{ \pm}, \eta\right)=2 R \bar{R} g\left(\xi, k_{ \pm}\right) .
$$

The relations (110) in the Appendix imply in general

$$
\begin{array}{r}
\boldsymbol{\eta}+2 R \bar{R} \boldsymbol{\xi}=-4 R \bar{R}\left[g\left(k_{-}, \xi\right) \boldsymbol{k}_{+}+g\left(k_{+}, \xi\right) \boldsymbol{k}_{-}\right] \\
R \overline{\boldsymbol{\chi}}+\bar{R} \boldsymbol{\chi}=4 R \bar{R}\left[g\left(k_{-}, \xi\right) \boldsymbol{k}_{+}-g\left(k_{+}, \xi\right) \boldsymbol{k}_{-}\right]
\end{array}
$$

from where some particular possibilities arise

1. Case with $g\left(k_{+}, \xi\right)=0$. In this situation one easily obtains

$$
g\left(k_{+}, \chi\right)=g\left(k_{+}, \eta\right)=0, \quad g(\chi, \bar{\chi})=-4 N R \bar{R}
$$

and also

$$
-(\boldsymbol{\eta}+2 R \bar{R} \boldsymbol{\xi})=R \overline{\boldsymbol{\chi}}+\bar{R} \boldsymbol{\chi}=4 R \bar{R} g\left(k_{-}, \xi\right) \boldsymbol{k}_{+} .
$$

2. Case $g\left(k_{+}, \xi\right)=g\left(k_{-}, \xi\right)=0$. In this subcase one obviously has, in addition to the above,

$$
g\left(k_{-}, \chi\right)=g\left(k_{-}, \eta\right)=0, \quad \boldsymbol{\eta}=-2 R \bar{R} \boldsymbol{\xi}, \quad R \overline{\boldsymbol{\chi}}+\bar{R} \boldsymbol{\chi}=0
$$

so that $\eta, \xi$ and $\chi$ are all eigenvectors of $t_{\mu \nu}$, the first two with eigenvalue $-R \bar{R} / 2$, the last with the opposite eigenvalue.

\section{$2.1 \quad \Lambda$-vacuum}

Throughout this paper we will assume that the spacetime satisfies the Einstein field equations for vacuum with a cosmological constant $\Lambda$, that is to say,

$$
R_{\alpha \beta}=\Lambda g_{\alpha \beta}
$$

where $R_{\alpha \beta}$ is the Ricci tensor. In that case we will say that the metric $g$ is $\Lambda$-vacuum. The Weyl curvature tensor of the spacetime is denoted by $C_{\alpha \beta \lambda \mu}$ and its (unique) Hodge dual by $C_{\alpha \beta \lambda \mu}^{\star}=\frac{1}{2} \eta_{\rho \sigma \lambda \mu} C_{\alpha \beta}{ }^{\rho \sigma}$. Then, the complex self-dual Weyl tensor is

$$
\mathcal{C}_{\alpha \beta \lambda \mu}=C_{\alpha \beta \lambda \mu}+i C_{\alpha \beta \lambda \mu}^{\star}, \quad \mathcal{C}_{\alpha \beta \lambda \mu}^{\star}=-i \mathcal{C}_{\alpha \beta \lambda \mu} .
$$

Under assumption (12) one has that the Riemann tensor becomes

$$
R_{\alpha \beta \lambda \mu}=C_{\alpha \beta \lambda \mu}+\frac{\Lambda}{3}\left(g_{\alpha \lambda} g_{\beta \mu}-g_{\alpha \mu} g_{\beta \lambda}\right)
$$

so that

$$
\nabla^{\alpha} \mathcal{C}_{\alpha \beta \lambda \mu}=0
$$

and the standard property of Killing vectors $\nabla_{\beta} \nabla_{\lambda} \xi_{\mu}=\xi^{\alpha} R_{\alpha \beta \lambda \mu}$ can be appropriately rewritten in the language of complex self-dual objects as

$$
\nabla_{\mu} \mathcal{F}_{\alpha \beta}=\xi^{\nu}\left(\mathcal{C}_{\nu \mu \alpha \beta}+\frac{4 \Lambda}{3} \mathcal{I}_{\nu \mu \alpha \beta}\right)
$$


where

$$
\mathcal{I}_{\alpha \beta \mu \nu}=\frac{1}{4}\left(g_{\alpha \mu} g_{\beta \nu}-g_{\alpha \nu} g_{\beta \mu}+i \eta_{\alpha \beta \mu \nu}\right)
$$

is the metric in the space of complex self-dual 2-forms, i.e. it satisfies $\mathcal{I}_{\alpha \beta \mu \nu} \mathcal{U}^{\mu \nu}=\mathcal{U}_{\alpha \beta}$ for any self-dual two form.

Taking the covariant derivative of the first in (5) one can obtain

$$
\nabla_{\mu} \chi_{\nu}=-\frac{\mathcal{F}^{2}}{4} g_{\mu \nu}-2 t_{\mu \nu}+2 \xi^{\rho} \xi^{\sigma} \mathcal{C}_{\rho \mu \sigma \nu}-\frac{2 \Lambda}{3}\left(N g_{\mu \nu}+\xi_{\mu} \xi_{\nu}\right)
$$

and therefore, in this situation the Ernst one-form is closed [40]

$$
\nabla_{[\mu} \chi_{\nu]}=0 .
$$

For completeness, one can also derive an expression for the covariant derivative of $\eta$, which after a calculation becomes

$$
\nabla_{\mu} \eta_{\nu}=-\frac{\overline{\mathcal{F}}^{2}}{4} \mathcal{F}_{\mu \nu}-\frac{\Lambda}{3}\left(\xi_{\mu} \chi_{\nu}+2 N \mathcal{F}_{\mu \nu}\right)+\xi^{\sigma} \bar{\chi}^{\rho} \mathcal{C}_{\sigma \mu \rho \nu}+\text { c.c. }
$$

Immediate consequences of (15) are

$$
\begin{aligned}
\nabla_{\mu} \mathcal{F}^{2} & =2 \xi^{\nu} \mathcal{F}^{\alpha \beta} \mathcal{C}_{\nu \mu \alpha \beta}+\frac{4 \Lambda}{3} \chi_{\mu}, \\
\nabla_{\alpha} \chi^{\alpha} & =-\mathcal{F}^{2}-2 \Lambda N \\
\nabla_{\mu} \nabla^{\mu} \mathcal{F}^{2} & =-\mathcal{C}_{\alpha \beta \mu \nu} \mathcal{F}^{\alpha \beta} \mathcal{F}^{\mu \nu}-\frac{4}{3} \Lambda \mathcal{F}^{2}-\frac{N}{2}\left(\mathcal{C}_{\alpha \beta \mu \nu} \mathcal{C}^{\alpha \beta \mu \nu}+\frac{16 \Lambda^{2}}{3}\right) .
\end{aligned}
$$

\section{$2.2 \quad$ Alignment of $\mathcal{C}$ and $\mathcal{F}$}

The self-dual $\mathcal{C}_{\alpha \beta \lambda \mu}$ defines an eigenvalue problem acting on self-dual 2 -forms. The content of this problem leads to the important Petrov classification of the Weyl tensor [45]. In the case under consideration, the spacetime has a distinguished self-dual 2-form, the Killing 2 -form $\mathcal{F}_{\mu \nu}$. It seems natural to ask what are the implications of $\mathcal{F}_{\mu \nu}$ being an eigen-2-form of $\mathcal{C}_{\alpha \beta \lambda \mu}$

$$
\mathcal{C}_{\alpha \beta \mu \nu} \mathcal{F}^{\alpha \beta} \propto \mathcal{F}_{\mu \nu}
$$

and this was the essential assumption in the several unique characterizations of the Kerr and other relative spacetimes given in references [30, 31, 32, 33].

The previous relation does not restrict the Petrov type of the spacetime in general. However, a particularly interesting case where that condition is achieved is by assuming

$$
\mathcal{C}_{\alpha \beta \mu \nu}=Q\left(\mathcal{F}_{\alpha \beta} \mathcal{F}_{\mu \nu}-\frac{1}{3} \mathcal{F}^{2} \mathcal{I}_{\alpha \beta \mu \nu}\right)
$$

which is actually the starting point in 30] - also [33]. We will assume this condition herein. If (22) holds, then at $p \in \mathcal{M}$

$$
k_{ \pm}^{\alpha} k_{ \pm}^{\mu} \mathcal{C}_{\alpha \beta \mu \nu}=\frac{2}{3} Q R^{2} k_{\beta}^{ \pm} k_{\nu}^{ \pm}
$$


showing that the real null eigenvectors of $\mathcal{F}_{\mu \nu}$ are multiple principal null directions of the Weyl tensor, so that the Petrov type is $\mathrm{D}$ if $\left.Q \mathcal{F}^{2}\right|_{p} \neq 0, \mathrm{~N}$ if $\left.\mathcal{F}^{2}\right|_{p}=0$ and $\left.Q\right|_{p} \neq 0$, or type 0 if $\left.Q\right|_{p}=0$.

Under the assumption (22) equations (15), (19) and (21) become, after using (5),

$$
\begin{aligned}
& \nabla_{\mu} \mathcal{F}_{\alpha \beta}=\frac{1}{2} Q \chi_{\mu} \mathcal{F}_{\alpha \beta}+\frac{1}{3}\left(4 \Lambda-Q \mathcal{F}^{2}\right) \xi^{\nu} \mathcal{I}_{\nu \mu \alpha \beta}, \\
& \nabla_{\mu} \mathcal{F}^{2}=\frac{2}{3}\left(Q \mathcal{F}^{2}+2 \Lambda\right) \chi_{\mu} \\
& \nabla_{\mu} \nabla^{\mu} \mathcal{F}^{2}=-\frac{2}{3} \mathcal{F}^{2}\left(Q \mathcal{F}^{2}+2 \Lambda\right)-\frac{N}{3}\left(Q^{2}\left(\mathcal{F}^{2}\right)^{2}+8 \Lambda^{2}\right) .
\end{aligned}
$$

Similarly, equations (16) and (18) become, respectively

$$
\begin{aligned}
& \nabla_{\mu} \chi_{\nu}=-\frac{\mathcal{F}^{2}}{4} g_{\mu \nu}-2 t_{\mu \nu}+\frac{Q}{2} \chi_{\mu} \chi_{\nu}+\frac{1}{6}\left(Q \mathcal{F}^{2}-4 \Lambda\right)\left(N g_{\mu \nu}+\xi_{\mu} \xi_{\nu}\right) \\
& \nabla_{\mu} \eta_{\nu}=-\frac{\overline{\mathcal{F}}^{2}}{4} \mathcal{F}_{\mu \nu}+\frac{1}{12}\left(\bar{Q} \overline{\mathcal{F}}^{2}-4 \Lambda\right)\left(\xi_{\mu} \chi_{\nu}+2 N \mathcal{F}_{\mu \nu}\right)+\frac{1}{2} Q \chi_{\mu} \eta_{\nu}+\text { c.c. }
\end{aligned}
$$

\section{Existence of Ernst potential and a second Killing vector}

In this section we will assume that the self-dual Killing form $\mathcal{F}_{\alpha \beta}$ is regular everywhere, i.e. $\mathcal{F}^{2} \neq 0$ everywhere on $\mathcal{M}$. This implies the existence of a smooth complex function $R: \mathcal{M} \longrightarrow \mathbb{C}$ satisfying $\mathcal{F}^{2}=-4 R^{2}$. Note that at each point $\pm R$ coincide with the eigenvalues introduced in Section 2 , and that there are two possible choices for the smooth function $R$, namely $R$ and $-R$. We assume from now on that one such choice has been made. Let $k=k_{+}$be a (real) null eigenvector field with eigenvalue $R$ and $\ell=k_{-}$a (real) null eigenvector field with eigenvalue $-R$. Being eigenvectors of multiplicity one, they can be both chosen to be smooth on $\mathcal{M}$ and, without loss of generality, as future directed and satisfying $g(k, \ell)=-1$. The freedom left in the choice is $k^{\prime} \longrightarrow A k, \ell^{\prime} \longrightarrow A^{-1} \ell$ with $A: \mathcal{M} \longrightarrow \mathbb{R}^{+}$smooth. Note that, had we chosen $-R$ instead of $R$, the null vector fields $\{k, \ell\}$ would have been interchanged.

Let us also introduce a complex one-form $P_{\alpha}$, a real one-form $q_{\alpha}$ and a self-dual two-form $\mathcal{W}_{\alpha \beta}$ by means of

$$
\chi_{\alpha} \stackrel{\text { def }}{=} 2 R P_{\alpha}, \quad \eta_{\mu} \stackrel{\text { def }}{=} 2 R \bar{R} q_{\mu}, \quad \mathcal{W}_{\alpha \beta} \stackrel{\text { def }}{=} \frac{1}{R} \mathcal{F}_{\alpha \beta} .
$$

These objects are all smooth and satisfy the following properties as a consequence of the 
expressions in Section 2

$$
\begin{array}{llll}
\mathcal{W}_{\mu \rho} \mathcal{W}_{\nu}{ }^{\rho}=-g_{\mu \nu}, & \mathcal{W}_{\alpha \beta} \mathcal{W}^{\alpha \beta}=-4, & \mathcal{W}_{\mu \rho} \overline{\mathcal{W}}^{\mu \rho}=0, & \\
P_{\alpha}=\xi^{\beta} \mathcal{W}_{\beta \alpha}, & P^{\alpha} \mathcal{W}_{\alpha \beta}=\xi_{\beta}, & \bar{P}^{\alpha} \mathcal{W}_{\alpha \beta}=q_{\beta}, & q^{\alpha} \mathcal{W}_{\alpha \beta}=\bar{P}_{\beta}, \\
\xi^{\alpha} P_{\alpha}=0, & q^{\alpha} P_{\alpha}=0, & P^{\alpha} P_{\alpha}=-q^{\alpha} q_{\alpha}=N, & q^{\mu} \xi_{\mu}=-P^{\mu} \bar{P}_{\mu}, \\
t_{\mu \nu}=\frac{R \bar{R}}{2} \mathcal{W}_{\mu \rho} \overline{\mathcal{W}}_{\nu}{ }^{\rho}, & P^{\alpha} t_{\alpha \beta}=-\frac{R \bar{R}}{2} \bar{P}_{\beta}, & \xi^{\alpha} t_{\alpha \beta}=-\frac{R \bar{R}}{2} q_{\beta}, & q^{\alpha} t_{\alpha \beta}=-\frac{R \bar{R}}{2} \xi_{\beta}, \\
\xi(R)=0, & £_{\xi} \mathcal{W}_{\mu \nu}=0, & {[\xi, P]=0,} & {[\xi, q]=0,}
\end{array}
$$

as well as

$$
\boldsymbol{\xi} \wedge \boldsymbol{q}=i \star(\boldsymbol{P} \wedge \overline{\boldsymbol{P}}) \quad \Longrightarrow \quad i \eta_{\alpha \beta \lambda \mu} \bar{P}^{\beta} \xi^{\lambda} P^{\mu}=N q_{\alpha}-\left(P_{\mu} \bar{P}^{\mu}\right) \xi_{\alpha}
$$

In combination with the null eigenvectors $k$ and $\ell$ we also have

$$
\begin{aligned}
t_{\alpha \beta} & =\frac{R \bar{R}}{2}\left(g_{\alpha \beta}+2 k_{\alpha} \ell_{\beta}+2 k_{\beta} \ell_{\alpha}\right), \\
\mathcal{W}_{\alpha \beta} & =W_{\alpha \beta}^{-}-i W_{\alpha \beta}^{+}, \quad \text { with } \quad W_{\alpha \beta}^{-}=-k_{\alpha} \ell_{\beta}+k_{\beta} \ell_{\alpha}, \quad W_{\alpha \beta}^{+}=\eta_{\alpha \beta \mu \nu} k^{\mu} \ell^{\nu} \\
W_{\alpha \beta}^{-} W^{-\alpha \beta} & =-W_{\alpha \beta}^{+} W^{+\alpha \beta}=-2, \quad W_{\alpha \beta}^{-} W^{+\alpha \beta}=0, \\
g(k, P) & =g(k, q)=g(k, \xi), \\
-g(\ell, P) & =g(\ell, q)=g(\ell, \xi), \\
\boldsymbol{\xi}+\boldsymbol{q} & =-2[g(\ell, \xi) \boldsymbol{k}+g(k, \xi) \ell] \\
\boldsymbol{P}+\overline{\boldsymbol{P}} & =2[g(\ell, \xi) \boldsymbol{k}-g(k, \xi) \ell] .
\end{aligned}
$$

In this section, it is useful to introduce a smooth function $J$ by

$$
Q \stackrel{\text { def }}{=} \frac{3 J}{R}-\frac{\Lambda}{R^{2}}
$$

where recall that $Q$ is the proportionality factor in (22). In terms of the new variables, equation (24) transforms into

$$
\nabla_{\mu} R=(2 J R-\Lambda) P_{\mu}
$$

which, inserted in (20), implies the following expression for the divergence of $P_{\mu}$

$$
\nabla_{\mu} P^{\mu}=2(R-J N) .
$$

More generally we have

Lemma 1. The tensors $\mathcal{W}_{\alpha \beta}, P_{\alpha}, q_{\alpha}$ and $\xi_{\alpha}$ satisfy the following equations

$$
\begin{aligned}
& \nabla_{\mu} \mathcal{W}_{\alpha \beta}=J\left(P_{\mu} \mathcal{W}_{\alpha \beta}+4 \xi^{\nu} \mathcal{I}_{\nu \mu \alpha \beta}\right) \\
& \nabla_{\mu} P_{\alpha}=\frac{R}{2} g_{\mu \alpha}-\frac{1}{R} t_{\mu \alpha}+J\left(P_{\mu} P_{\alpha}-N g_{\mu \alpha}-\xi_{\mu} \xi_{\alpha}\right) \\
& \nabla_{\mu} q_{\alpha}=\left(\frac{\bar{R}}{2}-N \bar{J}\right) \mathcal{W}_{\mu \alpha}+\left(\frac{R}{2}-N J\right) \overline{\mathcal{W}}_{\mu \alpha}+q_{\alpha}\left(J P_{\mu}+\overline{J P}_{\mu}\right)-\xi_{\mu}\left(\bar{J} P_{\alpha}+J \bar{P}_{\alpha}\right) \\
& \nabla_{\mu} \xi_{\alpha}=\frac{1}{2}\left(R \mathcal{W}_{\mu \alpha}+\bar{R} \overline{\mathcal{W}}_{\mu \alpha}\right)
\end{aligned}
$$


Proof: First note that (23) in terms of the new variables becomes

$$
\nabla_{\mu} \mathcal{F}_{\alpha \beta}=(3 J R-\Lambda) P_{\mu} \mathcal{W}_{\alpha \beta}+4 J R \xi^{\nu} \mathcal{I}_{\nu \mu \alpha \beta}
$$

which inserted into

$$
\nabla_{\mu} \mathcal{W}_{\alpha \beta}=\frac{1}{R} \nabla_{\mu} \mathcal{F}_{\alpha \beta}-\frac{1}{R^{2}} \mathcal{F}_{\alpha \beta} \nabla_{\mu} R
$$

gives (45). The second and third equations follow similarly from (26) and (27) after a straightforward calculation. The fourth is obvious from $\nabla_{\mu} \xi_{\alpha}=\frac{1}{2}\left(\mathcal{F}_{\mu \alpha}+\overline{\mathcal{F}}_{\mu \alpha}\right)$.

Lemma 2. The principal null directions $k$ and $\ell$ satisfy the following relations

$$
\begin{aligned}
& 2 \nabla_{\mu} k_{\beta}=k_{\beta}\left(J P_{\mu}+\overline{J P}_{\mu}-\ell^{\alpha} \nabla_{\mu} k_{\alpha}\right)+(J+\bar{J})\left[\xi_{\beta} k_{\mu}-g(\xi, k) g_{\beta \mu}\right]+i(J-\bar{J}) \xi^{\nu} k^{\alpha} \eta_{\nu \alpha \mu \beta}, \\
& 2 \nabla_{\mu} \ell_{\beta}=\ell_{\beta}\left(J P_{\mu}+\overline{J P}_{\mu}-k^{\alpha} \nabla_{\mu} \ell_{\alpha}\right)-(J+\bar{J})\left[\xi_{\beta} \ell_{\mu}-g(\xi, \ell) g_{\beta \mu}\right]-i(J-\bar{J}) \xi^{\nu} \ell^{\alpha} \eta_{\nu \alpha \mu \beta} .
\end{aligned}
$$

Proof: Taking the real part of (45) one derives

$2 \nabla_{\mu} W_{\alpha \beta}^{-}=\left(J P_{\mu}+\overline{J P}_{\mu}\right) W_{\alpha \beta}^{-}-i\left(J P_{\mu}-\overline{J P}_{\mu}\right) W_{\alpha \beta}^{+}+(J+\bar{J})\left(\xi_{\alpha} g_{\mu \beta}-\xi_{\beta} g_{\mu \alpha}\right)+i(J-\bar{J}) \xi^{\nu} \eta_{\nu \mu \alpha \beta}$

whose left-hand side equals by the definition (36)

$$
2\left(\ell_{\alpha} \nabla_{\mu} k_{\beta}+k_{\beta} \nabla_{\mu} \ell_{\alpha}-k_{\alpha} \nabla_{\mu} \ell_{\beta}+\ell_{\beta} \nabla_{\mu} k_{\alpha}\right) .
$$

Contracting then with $k^{\alpha}$, and with $\ell^{\alpha}$, gives the result directly.

Equation (46) implies directly that

$$
\nabla_{\alpha} P_{\beta}-\nabla_{\beta} P_{\alpha}=0
$$

so that the one-form $P_{\alpha}$ is closed. This will be used in the proof of the next Lemma.

Lemma 3. The Ernst one-form $\chi_{\alpha}$ (or equivalently $P_{\alpha}$ ) is non-zero almost everywhere and in fact vanishes only at points where $\xi$ vanishes. Moreover, $\xi$ is null at most on sets with empty interior and the function $J$ satisfies the equation

$$
\nabla_{\mu} J=J^{2} P_{\mu}
$$

Proof: From the first identity in (6) and $\mathcal{F}^{2} \neq 0$ it follows that $\chi_{\mu}$ vanishes at one point if and only if $\xi$ vanishes there. In particular $\chi_{\alpha}\left(\right.$ or $\left.P_{\alpha}\right)$ cannot vanish on any non-empty open set. For the second statement, assume that $\xi$ were null on a non-empty open set $V \subset \mathcal{M}$, i.e. $N=0$ on $V$. The last expression in (5) would imply $\chi^{\alpha} \chi_{\alpha}=0$ which combined with 
(7) would lead to $\chi_{\alpha}=i \omega_{\alpha}$ being null so that necessarily $\chi_{\mu}=2 A \xi_{\mu}$ for some function $A$ such that $A=-\bar{A}$. But then the first in (6) would provide

$$
2 A \xi^{\rho} \mathcal{F}_{\rho \mu}=A \chi_{\mu}=2 A^{2} \xi_{\mu}=2 R^{2} \xi_{\mu}
$$

so that $A= \pm R$, that is to say $\chi_{\mu}= \pm 2 R \xi_{\mu}$ with $R=-\bar{R}$. Thus $P_{\mu}= \pm \xi_{\mu}$ and the left-hand side of (44) is real while its right-hand side is purely imaginary (since $N=0$ ). So $R=0$ and thus $\chi_{\mu}$ would vanish on $V$, a contradiction.

Using $\mathcal{F}^{2}=-4 R^{2}$ and (43) equation (25) transforms into

$$
P_{\mu} \nabla^{\mu} J=J^{2} N
$$

Taking the exterior derivative in (43) and using the fact that $P_{\alpha}$ is closed one derives the existence of a smooth function $G: V_{0} \longrightarrow \mathbb{C}$ on the open dense set $V_{0}:=\left\{p \in \mathcal{M} ;\left.\boldsymbol{\chi}\right|_{p} \neq 0\right\}$ satisfying $\nabla_{\mu} J=G P_{\mu}$. Inserting this into (53) implies $N\left(G-J^{2}\right)=0$. Since $N$ vanishes almost nowhere, we conclude $G=J^{2}$ on $V_{0}$ and consequently (52) holds on the open, dense set $V_{0}$. Both the right- and the left-hand sides in this equation are smooth one-forms on $\mathcal{M}$. Since they agree on an open, dense set, they agree everywhere.

The next lemma shows that $P_{\mu}$ and $\chi_{\mu}$ are not only closed but in fact exact, and provides explicit expressions for $J, R$ and the Ernst potential $\chi$ satisfying $\chi_{\alpha}=\nabla_{\alpha} \chi$.

Lemma 4. Assume that $Q$ is not identically zero or $\Lambda \neq 0$. Then, the one-form $P_{\alpha}$ is exact on $\mathcal{M}$ and the complex function $P: \mathcal{M} \longrightarrow \mathbb{C}$ satisfying $P_{\alpha}=\nabla_{\alpha} P$ can be chosen to be non-zero everywhere. Moreover, there exist smooth complex functions $j, r, x$ defined on $\mathbb{C} \backslash\{0\}$ such that $J$ and $R$ satisfy $J=j \circ P, R=r \circ P$ and $\chi \stackrel{\text { def }}{=} x \circ P$ satisfies (i) $\nabla_{\alpha} \chi=\chi_{\alpha}$ and (ii) $\chi+\bar{\chi}=2 N$ (in particular, the Ernst one-form is exact). Moreover, $\{j, r, x\}$ belong to one of the two following classes,

$$
\begin{array}{ll}
\text { (A) } j(\zeta)=0, \quad r(\zeta)=-\Lambda \zeta, \quad x(\zeta)=c-\Lambda \zeta^{2}, \quad c \in \mathbb{R}, \quad \Lambda \neq 0 . \\
\text { (B) } j(\zeta)=-\frac{1}{\zeta}, \quad r(\zeta)=\frac{b}{2 \zeta^{2}}-\frac{\Lambda}{3} \zeta, \quad x(\zeta)=c-\frac{b}{\zeta}-\frac{\Lambda}{3} \zeta^{2}, \quad b=b_{1}+i b_{2}, \quad b_{1}, b_{2}, c \in \mathbb{R} .
\end{array}
$$

Proof: Assume first that $J$ vanishes everywhere on $\mathcal{M}$. Then, $\Lambda \neq 0$ (otherwise we would also have $Q=0$ against hypothesis). Equation (43) implies $P_{\alpha}=-\nabla_{\alpha}\left(\frac{R}{\Lambda}\right)$, and hence $P \stackrel{\text { def }}{=}-\frac{R}{\Lambda}$ satisfies $\nabla_{\alpha} P=P_{\alpha}$ and vanishes nowhere, as claimed. From $\chi_{\alpha}=2 R P_{\alpha}$ it follows $\chi_{\alpha}=-\frac{1}{\Lambda} \nabla_{\alpha} R^{2}$ which implies $\chi_{\alpha}=\nabla_{\alpha} \chi$ for $\chi=-\frac{R^{2}}{\Lambda}+c$ for a constant $c$ which can be taken to be real without loss of generality. Moreover, from (7) it follows that $c$ can always be adjusted so that $\chi+\bar{\chi}=2 N$. This proves the Lemma for class (A).

Assume now that $J$ is not identically zero. We first show that $J$ vanishes nowhere. Let $V_{1} \stackrel{\text { def }}{=}\{p \in \mathcal{M} ; J(p) \neq 0\}$ and assume that $V_{1} \neq \mathcal{M}$. Let $p$ be a point in the topological boundary $\partial V_{1}$. Sufficiently near $p, P_{\alpha}$ is exact $P_{\alpha}=\nabla_{\alpha} P$ for some smooth function $P$. Since $P_{\mu}$ vanishes if and only if $\xi_{\mu}$ vanishes and the set of fixed points of a Killing vector is at 
least of co-dimension two, $p$ can be chosen so that $\left.P_{\mu}\right|_{p} \neq 0$. Restricting the neighbourhood of $p$ if necessary we can assume $P_{\mu}$ nowhere zero there and hence there exists a smooth complex function $j$ defined on a neighbourhood of $P_{0} \stackrel{\text { def }}{=} P(p) \in \mathbb{C}$ such that $J=j \circ P$ and $j^{\prime}=j^{2}$ (this follows from (52)). Uniqueness of solutions of ODE implies $j \equiv 0$ in a neighbourhood of $p$, against hypothesis. Thus $V_{1}=\mathcal{M}$ and $J \neq 0$ everywhere. We can define $P=-\frac{1}{J}$ which satisfies $\nabla_{\alpha} P=P_{\alpha}$ (hence $P_{\alpha}$ is exact) and vanishes nowhere.

Equation (43) becomes

$$
\nabla_{\alpha} R=-\left(\frac{2 R}{P}+\Lambda\right) \nabla_{\alpha} P
$$

which integrates to $R=r \circ P$ with

$$
r=\frac{b}{2 \zeta^{2}}-\frac{\Lambda}{3} \zeta, \quad b \in \mathbb{C} .
$$

Finally, $\chi_{\alpha}=2 R P_{\alpha}$ reads

$$
\chi_{\alpha}=-\nabla_{\alpha}\left(\frac{b}{P}+\frac{\Lambda}{3} P^{2}\right)
$$

which proves that $\chi_{\alpha}$ is exact $\chi_{\alpha}=\nabla_{\alpha} \chi$ with $\chi=x \circ P$ and $x: \mathbb{C} \backslash\{0\} \rightarrow \mathbb{C}$ given by $x(\zeta)=c-b / \zeta-(\Lambda / 3) \zeta^{2}$, where $c$ is an arbitrary constant. Again without loss of generality $c$ can be chosen to be real and adjusted so that $\chi+\bar{\chi}=2 N$.

Remark: In the rest of the paper all results will split into the two cases (A) and (B) of this theorem. We will simply write (A) and (B) without further mention, e.g. in Proposition 1 below.

The complex function $P$ will be decomposed in real and imaginary parts as

$$
P=y+i Z
$$

where $y, Z: \mathcal{M} \rightarrow \mathbb{R}$ are smooth. The condition $P_{\alpha} P^{\alpha}=N$ (31) imposes

$$
\nabla_{\alpha} y \nabla^{\alpha} y-\nabla_{\alpha} Z \nabla^{\alpha} Z=N \quad \nabla_{\alpha} y \nabla^{\alpha} Z=0 .
$$

From $g(\xi, P)=g(q, P)=0$ and $g(k, P)=g(\xi, k)(\underline{38})$ and taking real and imaginary parts one has

$$
\xi(y)=0, \quad \xi(Z)=0, \quad q(y)=0, \quad q(Z)=0, \quad k(y)=g(\xi, k), \quad k(Z)=0 .
$$

From (47) and the formulas for case (A) in Lemma 4 one sees that, in this case, $\nabla_{(\mu} q_{\nu)}=$ 0 , so that $q$ is another Killing vector field on $\mathcal{M}$. The existence of a second Killing vector is proven in general in the next proposition

Proposition 1. The following vector fields 
(A) $\varsigma=A \xi+C q$,

(B) $\varsigma=\left(A-C P^{2}-C \bar{P}^{2}\right) \xi+2 C P \bar{P} q$.

are Killing vectors on $(\mathcal{M}, g)$ for arbitrary real constants $A$ and $C$. Moreover, any two vector fields in this class commute.

Proof: Let $\varsigma=F \xi+G q$ for some real functions $F$ and $G$ to be determined. A direct calculation using (47) and that $\xi$ is Killing provides

$$
\begin{aligned}
\nabla_{\mu} \varsigma_{\nu}+\nabla_{\nu} \varsigma_{\mu}= & \xi_{\mu}\left(\nabla_{\nu} F-G \bar{J} P_{\nu}-G J \bar{P}_{\nu}\right)+\xi_{\nu}\left(\nabla_{\mu} F-G \bar{J} P_{\mu}-G J \bar{P}_{\mu}\right) \\
& +q_{\mu}\left(\nabla_{\nu} G+G J P_{\nu}+G \overline{J P} \bar{P}_{\nu}\right)+q_{\nu}\left(\nabla_{\mu} G+G J P_{\mu}+G \overline{J P}{ }_{\mu}\right)
\end{aligned}
$$

so that $\varsigma$ is a Killing vector provided the following two equations are satisfied

$$
\nabla_{\mu} F=G \bar{J} P_{\mu}+G J \bar{P}_{\mu}, \quad \nabla_{\mu} G=-G J P_{\mu}-G \overline{J P}_{\mu} .
$$

Using the formulas in Lemma 4 we have that their general solution is $F=A, G=C$, where $A$ and $C$ are real constants in case (A). For case (B), the second equation integrates easily to

$$
G=2 C P \bar{P}
$$

and then the first can be solved to give

$$
F=-C\left(P^{2}+\bar{P}^{2}\right)+A .
$$

The commutation follows directly from (33) and $\xi(P)=\xi^{\mu} P_{\mu}=0$.

Remark. In the case of vanishing cosmological constant and assuming that the Killing vector $\xi$ is timelike so that it makes sense to pass locally to the quotient manifold defined by the orbits of $\xi$, a vector field equivalent to the one in case (B) of this Proposition was introduced by Perjés [41] as a useful tool to characterize locally the vacuum strictly stationary spacetimes with vanishing Simon tensor. This vector field was shown to leave invariant the metric components [41, so that it is a Killing vector field of the spacetime, see also [44].

This Proposition provides two linearly independent Killing vectors except in some special situations where $q$ and $\xi$ happen to be co-linear. This leads to the analysis of the special cases arising from (34).

\subsection{Special cases}

From Eq. (34) we know that the set $\{\xi, q, P, \bar{P}\}$ constitutes a basis on the tangent spaces unless $P$ and $\bar{P}$ are collinear. These special cases are characterized by the condition $\boldsymbol{P} \wedge \overline{\boldsymbol{P}}=$ 0 . First note that at any $p \in \mathcal{M}$ and for any $a \in \mathbb{C}$,

$$
\left.\overline{\boldsymbol{P}}\right|_{p}=\left.\left.a \boldsymbol{P}\right|_{p} \quad \Longleftrightarrow q\right|_{p}=\left.a \xi\right|_{p},
$$


as a direct consequence of (30). Unless $\left.\xi\right|_{p}=0$ (and then $\left.q\right|_{p}=\left.\boldsymbol{P}\right|_{p}=0$ ) it follows that $a$ is real and actually $\boldsymbol{P}=a \overline{\boldsymbol{P}}=a^{2} \boldsymbol{P}$ at $p$ so that either $a= \pm 1$ or $\left.\boldsymbol{P}\right|_{p}=0$. Thus, $\left.\boldsymbol{P} \wedge \overline{\boldsymbol{P}}\right|_{p}=0$ is equivalent to either $\overline{\boldsymbol{P}}_{p}=\left.\epsilon \boldsymbol{P}\right|_{p}$ with $\epsilon= \pm 1$ or $\left.\boldsymbol{P}\right|_{p}=0$. In each case one has, respectively, $\left.q\right|_{p}=\left.\epsilon \xi\right|_{p}$ or $\left.q\right|_{p}=0$ and $\left.\xi\right|_{p}=0$, the last as a consequence of Lemma 3 . Conversely, if $\left.q\right|_{p}$ is proportional to $\left.\xi\right|_{p} \neq 0$, then necessarily $\left.q\right|_{p}= \pm\left.\xi\right|_{p}$ because of (56). Moreover, $\left.\xi\right|_{p}=0$ implies $\left.q\right|_{p}=0$ and $\left.\boldsymbol{P}\right|_{p}=0$.

If $\boldsymbol{P} \wedge \overline{\boldsymbol{P}}=0$ on an open connected set, then there is $\epsilon \in\{+1,-1\}$ such that $\boldsymbol{P}=\epsilon \overline{\boldsymbol{P}}$ everywhere because of Lemma 3 and we then have $q=\epsilon \xi$. Conversely, $\boldsymbol{\xi} \wedge \boldsymbol{q}=0$ on an open set implies $q=\epsilon \xi$ for $\epsilon \in\{-1,1\}$ constant and then $\boldsymbol{P}=\epsilon \overline{\boldsymbol{P}}$. From (44) it also follows

$$
R-\epsilon \bar{R}=N(J-\epsilon \bar{J})
$$

which, upon using the expressions in Lemma 4, becomes

$$
\begin{array}{ll}
\text { (A): } \quad y(\epsilon-1)-i Z(\epsilon+1)=0 \\
\text { (B): } \quad(\epsilon-1)\left(b_{1}-2 c y+\frac{4 \Lambda}{3} y^{3}\right)+i(\epsilon+1)\left(b_{2}-2 c Z-\frac{4 \Lambda}{3} Z^{3}\right)=0 .
\end{array}
$$

Further information can be obtained for each value of $\epsilon$ separately

1. $\left.\boldsymbol{P}\right|_{p} \neq 0$ purely imaginary $(\epsilon=-1)$ : From (40,41) and (38) one gets at $p$

$$
g(k, P)=g(k, q)=g(k, \xi)=g(\ell, P)=g(\ell, q)=g(\ell, \xi)=0
$$

so that $\langle\xi, P\rangle$ is at $p$ a plane orthogonal to the plane generated by the principal null directions $\langle k, \ell\rangle$. One also has

$$
\left.2 \boldsymbol{\xi} \wedge \boldsymbol{P}\right|_{p}=\left.N(\overline{\mathcal{W}}-\mathcal{W})\right|_{p}=+\left.2 N i \boldsymbol{W}^{+}\right|_{p}
$$

2. $\left.\boldsymbol{P}\right|_{p} \neq 0$ real $(\epsilon=1)$ : From (40,41) and (38,39) one gets

$$
\left.\boldsymbol{P}\right|_{p}=\left.\overline{\boldsymbol{P}}\right|_{p}=\left.(g(\ell, \xi) \boldsymbol{k}-g(k, \xi) \ell)\right|_{p},\left.\quad \boldsymbol{\xi}\right|_{p}=\left.\boldsymbol{q}\right|_{p}=\left.(-g(\ell, \xi) \boldsymbol{k}-g(k, \xi) \boldsymbol{\ell})\right|_{p}
$$

so that $\langle\xi, P\rangle$ is at $p$ the plane generated by the principal null directions $\langle k, \ell\rangle$. One also has

$$
\left.2 \boldsymbol{\xi} \wedge \boldsymbol{P}\right|_{p}=\left.2 N \boldsymbol{k} \wedge \boldsymbol{\ell}\right|_{p}=-\left.N(\boldsymbol{W}+\overline{\mathcal{W}})\right|_{p}=-\left.2 N \boldsymbol{W}^{-}\right|_{p}
$$

3. If $\left.\boldsymbol{P}\right|_{p}=0$, all equations (60)-(63) hold trivially as $\left.\xi\right|_{p}=0$ and $\left.q\right|_{p}=0$ too.

\section{Solving the field equations}

Equation (46) will be the key for the integration of the field equations. This integration will proceed by identifying a natural Riemannian submersion and integrating the equations first on the quotient space and then going up into the total space. In the generic case, namely when the Killing vector $\xi$ is not simultaneously orthogonal to both null eigenvectors $k$ and 
$\ell$ and when $d Z \neq 0$, we will be able to identify enough structure in the quotient so that its geometry can be fully determined. In the special cases when either $d Z=0$ or when $\xi$ is orthogonal to both null eigenvectors, the problem will become harder and the fundamental equations for Riemannian submersions will have to be used. In fact, different submersions will have to be introduced in order to deal, respectively, with the cases (i) $d Z=0$ and (ii) $\xi$ orthogonal to both $k$ and $\ell$. In subsection 4.1 we identify the conformal rescaling in the spacetime that will allow is to define the Riemannian submersions in the following subsections. In subsection 4.2 we assume that $\xi$ and $k$ are not orthogonal and introduce a two-dimensional distribution in the spacetime which is proven to be integrable and to define a Riemannian submersion. The geometry of this Riemannian submersion is analyzed in detail with the additional assumption that $d Z \neq 0$. In subsection 4.3 we study this distribution in the case when $d Z=0$ and we also introduce a second distribution capable of dealing with the situation when $\xi$ is simultaneously orthogonal to $k$ and $\ell$. This second distribution is also shown to be two-dimensional and integrable and to define a Riemannian submersion. Despite the very different nature of both distributions, we introduce a notation that allows us to work with them simultaneously. Once the geometry of the Riemannian submersions is understood, we proceed by integrating up the field equations and determining the most general class of spacetime metrics satisfying our characterization hypotheses. This is done in subsection 4.4 where the main results of this section are stated and proved. Theorem 2 determines the spacetime metric when the Killing vector is not orthogonal to the eigenspace of the self-dual Killing form and Theorem 3 is the analogous result when $\xi$ is simultaneously orthogonal to $k$ and $\ell$.

\subsection{Conformal rescaling}

Let us first recall that a Riemannian submersion between two semi-Riemannian manifolds (of arbitrary signature) $(\mathcal{M}, g)$ and $(S, h)$ is a smooth surjective map $\pi: \mathcal{M} \rightarrow S$ of rank $=\operatorname{dim}(S)$ such that, for all $p \in S$ and all $q \in V_{p} \stackrel{\text { def }}{=} \pi^{-1}(p)$ the tangent space $T_{q} \mathcal{M}$ admits a direct sum decomposition $T_{q} \mathcal{M}=T_{q} V_{p} \oplus N_{q} V_{p}$, where $T_{q} V_{p}$ is the tangent space of $V_{p}$ (this is a submanifold because $\pi$ is of maximum rank) and $N_{q} V_{p}=\left(T_{q} V_{p}\right)^{\perp}$ (orthogonal with respect to the metric $g$ ) satisfying the property that $\pi_{\star}: N_{q} V_{p} \longrightarrow T_{p} S$ is an isometry. For details on Riemannian submersions see [39].

Consider now an integrable distribution $\mathcal{D}$ in $\mathcal{M}$ of dimension $m$ and assume that at each point $p \in \mathcal{M}$ the induced metric at $\mathcal{D}_{p} \subset T_{p} \mathcal{M}$ is non-degenerate. Let $\left\{\mathcal{L}_{\alpha}\right\}$ be the collection of maximal integrable manifolds of the distributions (which exists by the Frobenius theorem). This collection defines a foliation in $\mathcal{M}$ (as usual, each integrable manifold is called a "leaf"). For any subset $A \subset \mathcal{M}$ we define an equivalence relation: $p_{1}, p_{2} \in A$ are related $p_{1} \sim p_{2}$ if and only if the there exists a smooth path $\gamma$ from $p_{1}$ to $p_{2}$ fully contained in $A$ with tangent vector $\gamma^{\prime}$ everywhere tangent to $\mathcal{D}$. The quotient space will be denoted by $A / \sim$.

We can construct the orthogonal projector to $\mathcal{D}_{p}$, i.e. the one-one tensor $h^{\alpha}{ }_{\beta}$ satisfying $\left.h\right|_{\mathcal{D}_{p}}=0$ and $\left.h\right|_{\mathcal{D}_{p}^{\perp}}=\left.\mathrm{Id}\right|_{\mathcal{D}_{p}^{\perp}}$. Let $h_{\alpha \beta}=g_{\alpha \mu} h_{\beta}^{\mu}$ (which is well-known to be symmetric) and let $X$ be an arbitrary vector field on $\mathcal{M}$ tangent to $\mathcal{D}$ (i.e. $\left.X\right|_{p} \in \mathcal{D}_{p}, \forall p \in \mathcal{M}$ ). Then the 
following result is well-known (cf. Theorem 1.2.1 in [19])

Lemma 5. If $£_{X} h_{\alpha \beta}=0$ for all vector fields $X$ tangent to $\mathcal{D}$, then for any $p \in \mathcal{M}$ there exists an open connected neighbourhood $U_{p}$ of $p$ such that $U_{p} / \sim$ is a smooth manifold and the projection $\pi: U_{p} \rightarrow U_{p} / \sim$ is a Riemannian submersion.

The condition for $£_{X} h_{\alpha \beta}$ can be rewritten in terms of covariant derivatives of $X_{\alpha}$ as follows

Lemma 6. For any vector field $X$ tangent to $\mathcal{D}$ the following expression holds

$$
£_{X} h_{\alpha \beta}=h_{\alpha}^{\mu} h_{\beta}^{\nu}\left(\nabla_{\mu} X_{\nu}+\nabla_{\nu} X_{\mu}\right) .
$$

Proof: Let $Y$ be any vector field tangent to the distribution $\mathcal{D}$. It follows that $£_{X} Y$ is also tangent to the distribution. Let $V_{\alpha}$ be any one-form normal to the distribution, then $£_{X} V_{\alpha}$ is also normal to the distribution because, for any tangent vector $Y$, we have

$$
\left(£_{X} V_{\alpha}\right) Y^{\alpha}=£_{X}\left(V_{\alpha} Y^{\alpha}\right)-V_{\alpha}[X, Y]^{\alpha}=0 .
$$

Also $\left(£_{X} h_{\mu}^{\alpha}\right) V_{\alpha}=0$, because

$$
\left(£_{X} h_{\mu}^{\alpha}\right) V_{\alpha}=£_{X}\left(h_{\mu}^{\alpha} V_{\alpha}\right)-h_{\mu}^{\alpha} £_{X} V_{\alpha}=\left(\delta_{\mu}^{\alpha}-h_{\mu}^{\alpha}\right) £_{X} V_{\alpha}=0 .
$$

It it clear then that $\left(£_{X} h^{\mu}{ }_{\alpha}\right) h_{\mu \nu}=0$ (because its contraction in the index $\nu$ with a tangent vector or with a normal vector vanishes). Using $h_{\alpha \beta}=h_{\alpha}^{\mu} h_{\beta}^{\nu} g_{\mu \nu}$,

$$
£_{X} h_{\alpha \beta}=£_{X}\left(h^{\mu}{ }_{\alpha} h_{\beta}^{\nu} g_{\mu \nu}\right)=h_{\alpha}^{\mu} h_{\beta}^{\nu} £_{X} g_{\mu \nu}=h_{\alpha}^{\mu} h_{\beta}^{\nu}\left(\nabla_{\mu} X_{\nu}+\nabla_{\nu} X_{\mu}\right) \text {. }
$$

The Riemannian submersions we will define involve a suitable conformal rescaling of $g$. We start with the following Lemma concerning the principal null direction $k$.

Lemma 7. Let $\mathcal{D}$ be an integrable distribution in $\mathcal{M}$ such that $\left.k\right|_{p} \in \mathcal{D}_{p} \forall p \in \mathcal{M}$ and let $h_{\alpha \beta}$ be the corresponding projector. Then

$$
\begin{aligned}
h_{\mu}^{\alpha} h_{\nu}^{\beta}\left(\nabla_{\alpha} k_{\beta}-\nabla_{\beta} k_{\alpha}\right) & =i(J-\bar{J}) \xi^{\sigma} k^{\rho} \eta_{\sigma \rho \alpha \beta} h_{\mu}^{\alpha} h_{\nu}^{\beta}, \\
£_{k} h_{\alpha \beta} & =-g(\xi, k)(J+\bar{J}) h_{\alpha \beta} .
\end{aligned}
$$

Moreover, if $g(\xi, k) \neq 0$ everywhere then $k^{\alpha} \nabla_{\alpha} k_{\beta}=\frac{k(g(\xi, k))}{g(\xi, k)} k_{\beta}$.

Proof. Projecting (50) and using the fact that $h^{\alpha}{ }_{\beta} k_{\alpha}=0$ one gets

$$
2 h_{\mu}^{\alpha} h_{\nu}^{\beta} \nabla_{\alpha} k_{\beta}=-g(\xi, k)(J+\bar{J}) h_{\mu \nu}+i(J-\bar{J}) \xi^{\sigma} k^{\rho} \eta_{\sigma \rho \alpha \beta} h_{\mu}^{\alpha} h_{\nu}^{\beta} .
$$

The symmetric and anti-symmetric part of this relation give (65) and (64), respectively. For the last statement, (50) gives $2 k^{\alpha} \nabla_{\alpha} k_{\beta}=-\left(\ell^{\rho} k^{\sigma} \nabla_{\sigma} k_{\rho}\right) k_{\beta}$. Contracting with $\xi^{\beta}$ fixes

$$
-\ell^{\rho} k^{\sigma} \nabla_{\sigma} k_{\rho}=\frac{2 k(g(\xi, k))}{g(\xi, k)} .
$$


This Lemma suggests the metric transformation required in order to define a Riemannian submersion. Let $s: \mathbb{C} \backslash\{0\} \rightarrow \mathbb{C} \backslash\{0\}$ be a solution of the ODE $s^{\prime}=-s j$ and define $S \stackrel{\text { def }}{=} s \circ P$ and $\Omega \stackrel{\text { def }}{=} S \bar{S}$. The latter is a positive real function satisfying

$$
\nabla_{\alpha} \Omega=-\Omega\left(J P_{\alpha}+\overline{J P}_{\alpha}\right) .
$$

The conformally related metric

$$
\hat{g}_{\alpha \beta} \stackrel{\text { def }}{=} \frac{1}{\Omega} g_{\alpha \beta}
$$

will play a fundamental role in the integration of the field equations. Without loss of generality we choose $s$ (and hence $\Omega$ ) as follows in each of the classes defined in Lemma 4:
(A) $s(\zeta)=1, \quad \Omega=1$.
(B) $s(\zeta)=\zeta, \quad \Omega=y^{2}+Z^{2}$.

Lemma 8. Let $\mathcal{D}$ be a distribution satisfying the same hypothesis as in Lemma 7 . Let $\hat{h}_{\alpha \beta}$ be the corresponding projector with the spacetime metric $\hat{g}$. Then

$$
£_{k} \hat{h}_{\alpha \beta}=0 .
$$

Proof: First note that $k(\Omega)=-\Omega(J+\bar{J}) g(\xi, k)$. Since $\hat{h}_{\alpha \beta}=\frac{1}{\Omega} h_{\alpha \beta}$, it follows

$$
£_{k} \hat{h}_{\alpha \beta}=\frac{1}{\Omega}\left(£_{k} h_{\alpha \beta}-\frac{k(\Omega)}{\Omega} h_{\alpha \beta}\right)=\frac{1}{\Omega}\left(£_{k} h_{\alpha \beta}+g(\xi, k)(J+\bar{J}) h_{\alpha \beta}\right)=0 .
$$

We adopt the convention that all spacetime indices are raised and lowered with the metric $g$, unless contrarily indicated. The covariant derivative with respect to $\hat{g}$ will be denoted by $\hat{\nabla}$. The standard transformation law for covariant derivatives under conformal rescaling allows us to obtain $\widehat{\nabla}_{\mu} P_{\alpha}$ from (46). The result is

$$
\widehat{\nabla}_{\mu} P_{\alpha}=\frac{R}{2} g_{\mu \alpha}-\frac{1}{R} t_{\mu \alpha}-J\left(\xi_{\mu} \xi_{\alpha}+\frac{N}{2} g_{\mu \alpha}\right)-\frac{\bar{J}}{2}\left(\bar{P}_{\mu} P_{\alpha}+P_{\mu} \bar{P}_{\alpha}-g_{\mu \alpha} P_{\nu} \bar{P}^{\nu}\right)
$$

whose imaginary part implies the following equation for $Z$ :

$$
\begin{aligned}
2 i \widehat{\nabla}_{\mu} \widehat{\nabla}_{\alpha} Z= & (R-\bar{R})\left(\frac{1}{2} g_{\mu \alpha}+\frac{1}{R \bar{R}} t_{\mu \alpha}\right)+ \\
& +(J-\bar{J})\left(-\xi_{\mu} \xi_{\alpha}+\nabla_{\mu} y \nabla_{\alpha} y+\nabla_{\mu} Z \nabla_{\alpha} Z-g_{\mu \alpha}\left(N+\nabla_{\nu} Z \nabla^{\nu} Z\right)\right) .
\end{aligned}
$$




\subsection{Riemannian submersion in the generic case}

In this subsection we assume that $g(\xi, k) \neq 0$ and introduce a distribution shown to be a Riemannian submersion. Several of its properties, required later, are stated and proven. We start will the following Proposition.

Proposition 2. Assume that $\xi$ satisfies $g(\xi, k) \neq 0$ everywhere and define $\mathcal{D}_{p}=\left.\operatorname{span}\{\xi, k\}\right|_{p}$ $\subset T_{p} \mathcal{M}$. This collection of vector subspaces defines a two-dimensional integrable distribution with timelike leaves. Let $h_{\alpha}^{\mu}$ be the corresponding orthogonal projector and $\hat{h}_{\alpha \beta}=\hat{g}_{\mu \alpha} h_{\alpha}^{\mu}$. Then, for any vector field $X$ tangent to the distribution we have $£_{X} \hat{h}_{\alpha \beta}=0$ and the following equation holds

$$
h^{\mu}{ }_{\nu} h_{\beta}^{\alpha} \widehat{\nabla}_{\mu} \widehat{\nabla}_{\alpha} Z=U \hat{h}_{\nu \beta}, \quad \text { with } \quad U \stackrel{\text { def }}{=} \frac{\Omega}{2 i}(R-\bar{R}-N(J-\bar{J})) .
$$

Remark: Substituting the explicit expressions in classes (A) and (B) defined in Lemma 4, it follows that $U=u \circ Z$, where $u: \mathbb{R} \backslash\{0\} \rightarrow \mathbb{R}$ is given by

(A) $u=-\Lambda \zeta$.

(B) $u=\frac{b_{2}}{2}-c \zeta-\frac{2 \Lambda}{3} \zeta^{3}$.

Proof: The condition $g(\xi, k) \neq 0$ implies that $\xi$ and $k$ are linearly independent everywhere, so that $\mathcal{D}_{p}$ is two-dimensional. To show that it is integrable it suffices to show that $\left.[\xi, k]\right|_{p} \in \mathcal{D}_{p}$, but this is immediate because $k$ is a simple eigenvector of a tensor $\mathcal{F}_{\alpha \beta}$ which is invariant under $\xi$ and hence $[\xi, k] \propto k$. The induced metric on $\mathcal{D}_{p}$ is non-degenerate because clearly no linear combination of $\xi$ and $k$ can be orthogonal to $\xi$ and $k$ simultaneously. Being non-degenerate, it is obviously timelike because it contains a null direction. For the statement $£_{X} \hat{h}_{\alpha \beta}=0$, it suffices to check that $£_{\xi} \hat{h}_{\alpha \beta}=0$ and $£_{k} \hat{h}_{\alpha \beta}=0$. The first is immediate from the fact that $\xi$ is a Killing vector for $\hat{g}$, due to $\xi(\Omega)=0$, and the second has been proven in Lemma 8.

In order to establish (69), we define $V_{\alpha} \stackrel{\text { def }}{=} h^{\mu}{ }_{\alpha} \nabla_{\mu} y$. Note that $h^{\mu}{ }_{\alpha} \nabla_{\mu} Z=\nabla_{\alpha} Z$ because of (55). From (35) it follows $t_{\mu \alpha} h_{\nu}^{\nu} h_{\beta}^{\alpha}=\frac{R \bar{R}}{2} h_{\nu \alpha}$ and (68) implies

$2 i h^{\mu}{ }_{\nu} h_{\beta}^{\alpha} \widehat{\nabla}_{\mu} \widehat{\nabla}_{\alpha} Z=(R-\bar{R}-N(J-\bar{J})) h_{\nu \beta}+(J-\bar{J})\left(V_{\nu} V_{\beta}+\nabla_{\nu} Z \nabla_{\beta} Z-h_{\nu \beta} \nabla_{\rho} Z \nabla^{\rho} Z\right)$.

Since $h_{\nu \beta}=\Omega \hat{h}_{\nu \beta}$ it only remains to show that $V_{\nu} V_{\beta}+\nabla_{\nu} Z \nabla_{\beta} Z-h_{\nu \beta} \nabla_{\rho} Z \nabla^{\rho} Z$ vanishes. From (54) one has $V_{\alpha} \nabla^{\alpha} Z=0$. Moreover, it is immediate to check that the projector $h_{\alpha \beta}$ can be written as

$$
h_{\alpha \beta}=g_{\alpha \beta}-\frac{N}{g(\xi, k)^{2}} k_{\alpha} k_{\beta}-\frac{1}{g(\xi, k)}\left(k_{\alpha} \xi_{\beta}+k_{\beta} \xi_{\alpha}\right)
$$

and hence, using (55),

$$
\begin{aligned}
V_{\alpha} V^{\alpha} & =h^{\alpha \beta} \nabla_{\alpha} y \nabla_{\beta} y=\left(g^{\alpha \beta}-\frac{N}{g(\xi, k)^{2}} k^{\alpha} k^{\beta}-\frac{1}{g(\xi, k)}\left(k^{\alpha} \xi^{\beta}+k^{\beta} \xi^{\alpha}\right)\right) \nabla_{\alpha} y \nabla_{\beta} y= \\
& =\nabla_{\alpha} y \nabla^{\alpha} y-N=\nabla_{\alpha} Z \nabla^{\alpha} Z
\end{aligned}
$$


The claim now follows from the fact that $\mathcal{D}_{p}^{\perp}$ is two-dimensional.

The direct sum decomposition $T_{p} \mathcal{M}=\mathcal{D}_{p} \oplus \mathcal{D}_{p}^{\perp}$ allows to decompose any vector $X$ in $T_{p} \mathcal{M}$ as $X=X^{\mathcal{V}}+X^{\mathcal{H}}$, where $\mathcal{V}$ stands for "vertical" (i.e. along $\mathcal{D}_{p}$ ) and $\mathcal{H}$ for "horizontal" (i.e. along $\mathcal{D}_{p}^{\perp}$ ). The following lemma relates the orientation of the quotient space to the orientation of the ambient spacetime.

Lemma 9. Assume that $g(\xi, k) \neq 0$ everywhere, define $\mathcal{D}_{p}=\left.\operatorname{span}\{\xi, k\}\right|_{p} \subset T_{p} \mathcal{M}$ and assume that $\mathcal{M} / \sim$ is a manifold. Then there exists a volume form $\hat{\boldsymbol{\eta}}$ on $\mathcal{M} / \sim$ such that $\hat{\eta}_{\mu \nu} \stackrel{\text { def }}{=} \pi^{\star}(\hat{\boldsymbol{\eta}})_{\mu \nu}$ satisfies

$$
\Omega^{-1} \eta_{\alpha \beta \rho \sigma} h_{\mu}^{\alpha} h_{\nu}^{\beta} k^{\rho} \ell^{\sigma}=\Omega^{-1} W_{\mu \nu}^{+}=\hat{\eta}_{\mu \nu} .
$$

In particular $h_{\mu}^{\alpha} h_{\nu}^{\beta}\left(\nabla_{\alpha} k_{\beta}-\nabla_{\beta} k_{\alpha}\right)=i g(\xi, k)(J-\bar{J}) \Omega \hat{\eta}_{\mu \nu}$.

Proof: The two-form $W_{\alpha \beta}^{+}=\eta_{\alpha \beta \rho \sigma} k^{\rho} \ell^{\sigma}$ is orthogonal to $k$ so that, from (70),

$$
W_{\alpha \beta}^{+} W_{\mu \nu}^{+} h^{\alpha \mu} h^{\beta \nu}=W_{\alpha \beta}^{+} W^{+\alpha \beta}=2,
$$

where in the last equality we used (37). Consequently the two-form $W_{\alpha \beta}^{+} h_{\mu}^{\alpha} h^{\beta}{ }_{\nu}$ vanishes nowhere. Let $\hat{\eta}$ be the volume-form on $\mathcal{M} / \sim$ such that $W_{\alpha \beta}^{+} h^{\alpha}{ }_{\mu} h^{\beta}{ }_{\nu}$ is proportional to $\hat{\eta}_{\mu \nu}=\pi^{\star}(\hat{\boldsymbol{\eta}})_{\mu \nu}$ with a positive proportionality factor (existence follows because both are two-forms on the two-dimensional vector space $\mathcal{D}_{p}^{\perp}$ and both are everywhere non-zero). Squaring

$$
W_{\alpha \beta}^{+} h_{\mu}^{\alpha} h_{\nu}^{\beta}=f \hat{\eta}_{\mu \nu}, \quad f>0
$$

with the metric $\hat{g}$, and using $\hat{\eta}_{\mu \nu} \hat{\eta}_{\rho \sigma} \hat{g}^{\mu \rho} \hat{g}^{\nu \sigma}=2\left(\hat{g}^{\alpha \beta}\right.$ is the inverse of $\hat{g}_{\alpha \beta}$ and not the tensor obtained by raising indices with $g^{\alpha \beta}$ ) we conclude $f^{2}=\Omega^{2}$ and hence $f=\Omega$, as claimed.

Expanding the vertical part of $\ell$ in the basis $\{\xi, k\}$ we find $\ell^{\mathcal{V}}=-\frac{1}{g(\xi, k)} \xi+h_{0} k$ where $h_{0}$ is a function whose explicit from does not concern us. It follows that

$$
\eta_{\alpha \beta \rho \sigma} h_{\mu}^{\alpha} h_{\nu}^{\beta} k^{\rho} \ell^{\sigma}=\frac{1}{g(\xi, k)} \eta_{\alpha \beta \rho \sigma} h_{\mu}^{\alpha} h_{\nu}^{\beta} \xi^{\rho} k^{\sigma}
$$

so that the last statement follows directly from (64) in Lemma 7.

Remark. This Lemma implies that, for any pair of horizontal vectors $X_{1}=X_{1}^{\mathcal{H}}$ and $X_{2}=$ $X_{2}^{\mathcal{H}}$ in $T_{p} \mathcal{M}$, the pair $\left\{\pi_{\star}\left(X_{1}\right), \pi_{\star}\left(X_{2}\right)\right\}$ is positively oriented if and only if $\left\{k, \ell, X_{1}, X_{2}\right\}$ is positively oriented in $T_{p} \mathcal{M}$, and also if and only if $\frac{1}{g(\xi, k)} \eta_{\alpha \beta \rho \sigma} X_{1}^{\alpha} X_{2}^{\beta} \xi^{\rho} k^{\sigma}>0$. This will be used below.

The function $Z$ is constant along the leaves of $\{\mathcal{D}\}$. Hence there exists a function $\hat{Z}$ such that $Z=\pi^{\star}(\hat{Z})$. Let $\star_{\hat{h}} d \hat{Z}$ be the Hodge dual of $d \hat{Z}$, in components

$$
\left(\star_{\hat{h}} d \hat{Z}\right)_{A}=\hat{\eta}_{A B} D^{B} \hat{Z}
$$

where $D$ is the covariant derivative of $\hat{h}$ and all indices $(A, B, \ldots)$ on $\mathcal{M} / \sim$ are raised and lowered with $\hat{h}_{A B}$. It is immediate that $\left\{\star_{\hat{h}} d \hat{Z}, d \hat{Z}\right\}$ is either identically zero or defines a positively oriented basis. 
Lemma 10. Under the same conditions as in Lemma 9, assume further that $d Z \neq 0$ everywhere. Then, the one-form $V_{\alpha} \stackrel{\text { def }}{=} h^{\beta}{ }_{\alpha} \nabla_{\beta}$ y is given by $V_{\alpha}=\pi_{\star}\left(\star_{\hat{h}} d \hat{Z}\right)_{\alpha}$.

Proof: We already know the properties $V_{\alpha} V^{\alpha}=\nabla_{\alpha} Z \nabla^{\alpha} Z$ and $V_{\alpha} \nabla^{\alpha} Z=0$. Since both $V_{\alpha}$ and $\pi_{\star}\left(\star_{\hat{h}} d \hat{Z}\right)_{\alpha}$ are horizontal, it must happen that $V_{\alpha}=\epsilon_{1} \pi_{\star}\left(\star_{\hat{h}} d \hat{Z}\right)_{\alpha}$, with $\epsilon_{1}= \pm 1$. To decide the sign, it is necessary to analyze the orientation of $\left\{\xi^{\alpha}, k^{\beta}, V^{\alpha}, \nabla^{\alpha} Z\right\}$. We compute $\eta_{\alpha \beta \mu \nu} V^{\alpha} \nabla^{\beta} Z \xi^{\mu} k^{\nu}=\eta_{\alpha \beta \mu \nu} \nabla^{\alpha} y \nabla^{\beta} Z \xi^{\mu} k^{\nu}=-\frac{i}{2} \eta_{\alpha \beta \mu \nu} k^{\alpha} \bar{P}^{\beta} \xi^{\mu} P^{\nu}=-\frac{1}{2}\left(N q_{\nu}-P_{\alpha} \bar{P}^{\alpha} \xi_{\nu}\right) k^{\nu}$

where in the last equality we have used (34). Since $q_{\nu} k^{\nu}=g(\xi, k)$ (cf. (40) $)$ and $N-P_{\alpha} \bar{P}^{\alpha}=$ $P_{\alpha}\left(P^{\alpha}-\bar{P}^{\alpha}\right)=-2 \nabla_{\alpha} Z \nabla^{\alpha} Z$ we conclude that

$$
\frac{1}{g(\xi, k)} \eta_{\alpha \beta \mu \nu} V^{\alpha} \nabla^{\beta} Z \xi^{\mu} k^{\nu}=\nabla_{\alpha} Z \nabla^{\alpha} Z \geq 0 .
$$

Hence, in view of (171), $\left\{V^{\alpha}, \nabla^{\alpha} Z, k^{\alpha}, \ell^{\alpha}\right\}$ is positively oriented provided $\nabla^{\alpha} Z \neq 0$. Since $\left\{\star_{\hat{h}} d \hat{Z}, d \hat{Z}\right\}$ is also positively oriented in the quotient, it follows that $\epsilon_{1}=1$.

\subsection{Riemannian submersions in the special cases}

The results in the previous subsection will be sufficient to determine fully the geometry of the quotient space $(\mathcal{M} / \sim, \hat{h})$ of the Riemannian submersion when $d Z \neq 0$. The key for this will be equation (69) in Proposition 2, However, when $Z$ is a constant this equation gives no information at all, so an alternative method must be used. In addition, when $\xi$ is orthogonal to both $k$ and $\ell$ the distribution $\operatorname{span}\{\xi, \ell\}$ in the previous subsection is no longer non-degenerate, and hence does not define a Riemannian submersion. In order to deal with this situation we need to introduce an alternative distribution and show that it defines a Riemannian submersion. Note that the situation $Z=$ const. corresponds precisely to the special case $P_{\alpha}=\bar{P}_{\alpha}$ in Subsection 3.1 while the case $g(\xi, k)=g(\xi, \ell)=0$ corresponds to the other special case $P_{\alpha}=-\bar{P}_{\alpha}$, see (41).

The next lemma introduces the second distribution and proves it to define a Riemannian submersion.

Lemma 11. Assume $g(\xi, k)=g(\xi, \ell)=0$ with $\xi \neq 0$ everywhere. Then the distribution $\mathcal{D}_{p}^{-} \stackrel{\text { def }}{=} \operatorname{span}\left\{\left.\xi\right|_{p},\left.\operatorname{grad} Z\right|_{p}\right\}$ with $(\operatorname{grad} Z)^{\alpha}=\nabla^{\alpha} Z$ is two-dimensional, spacelike and integrable. Let ${H^{\mu}}_{\alpha}$ be its orthogonal projector and $\hat{H}_{\alpha \beta} \stackrel{\text { def }}{=} \hat{g}_{\alpha \mu} H_{\alpha}^{\mu}$. Then,

$$
£_{\xi} \hat{H}_{\alpha \beta}=£_{\text {grad } z} \hat{H}_{\alpha \beta}=0 .
$$

Proof: From $P_{\alpha}=-\bar{P}_{\alpha}$ it follows $y=$ const and hence, using (54), $\nabla_{\alpha} Z \nabla^{\alpha} Z=\xi_{\alpha} \xi^{\alpha}=$ $-N>0$ (recall that $P_{\alpha}=i \nabla_{\alpha} Z$ can vanish only if $\xi$ vanishes and $\xi^{\alpha} \nabla_{\alpha} Z=0$ ). This proves that the distribution $\left\{\mathcal{D}^{-}\right\}$is two-dimensional and spacelike and, moreover, that the metric $g_{\mu \alpha}$ can be decomposed as

$$
g_{\mu \alpha}=-k_{\mu} \ell_{\alpha}-k_{\alpha} \ell_{\mu}-\frac{1}{N}\left(\nabla_{\mu} Z \nabla_{\alpha} Z+\xi_{\mu} \xi_{\alpha}\right)=H_{\mu \alpha}-\frac{1}{N}\left(\nabla_{\mu} Z \nabla_{\alpha} Z+\xi_{\mu} \xi_{\alpha}\right) .
$$


$\left\{\mathcal{D}^{-}\right\}$is also integrable because $[\xi \operatorname{grad} Z]=0$ as a consequence of $\xi$ being a Killing vector. $£_{\xi} H_{\alpha \beta}=0$ for the same reason. To show $£_{\operatorname{grad}} H_{\alpha \beta}=0$ we use Lemma 6. First note, from (68) and $H^{\mu}{ }_{\alpha} \nabla_{\mu} Z=H_{\alpha}^{\mu} \xi_{\nu}=0$,

$$
2 i H_{\nu}^{\mu} H_{\beta}^{\alpha} \hat{\nabla}_{\mu} \hat{\nabla}_{\alpha} Z=(R-\bar{R}) H_{\nu}^{\mu} H_{\beta}^{\alpha}\left(\frac{1}{2} g_{\mu \alpha}+\frac{1}{R \bar{R}} t_{\mu \alpha}\right)=0
$$

where in the last equality we used (35) so that

$$
\frac{1}{2} g_{\mu \alpha}+\frac{1}{R \bar{R}} t_{\mu \alpha}=g_{\mu \alpha}+k_{\mu} \ell_{\alpha}+k_{\alpha} \ell_{\mu}=-N^{-1}\left(\nabla_{\mu} Z \nabla_{\alpha} Z+\xi_{\mu} \xi_{\alpha}\right) .
$$

Applying now Lemma 6 (observe that $\left.(\operatorname{grad} Z)^{\alpha} \hat{g}_{\alpha \beta}=\Omega^{-1} \hat{\nabla}_{\beta} Z\right)$

$$
£_{\operatorname{grad} Z} \hat{H}_{\alpha \beta}=H_{\alpha}^{\mu} H_{\beta}^{\nu}\left(\hat{\nabla}_{\mu}\left(\Omega^{-1} \hat{\nabla}_{\nu} Z\right)+\hat{\nabla}_{\nu}\left(\Omega^{-1} \hat{\nabla}_{\mu} Z\right)\right)=\frac{2}{\Omega} H_{\alpha}^{\mu} H_{\beta}^{\nu} \hat{\nabla}_{\mu} \hat{\nabla}_{\nu} Z=0 .
$$

In order to deal with the special cases we exploit the relationship between the Riemann tensor on the quotient and suitable components of the Riemann tensor on the ambient manifold valid for any Riemannian submersion

Proposition 3 ([39]). Let $\pi:(\mathcal{M}, \hat{g}) \rightarrow(S, \hat{h})$ be a Riemannian submersion and denote by ${ }^{\hat{g}} R,{ }^{\hat{h}} R$ the respective Riemann tensors. Let $\{X, Y, W, Z\}$ be horizontal vector fields. Then

$$
\begin{aligned}
{ }^{\hat{h}} R\left(\pi_{\star}(X), \pi_{\star}(Y), \pi_{\star}(W), \pi_{\star}(Z)\right)= & \hat{g} R(X, Y, W, Z)+\frac{1}{2} \hat{g}\left([X, Y]^{\mathcal{V}},[W, Z]^{\mathcal{V}}\right)+ \\
& +\frac{1}{4} \hat{g}\left([X, W]^{\mathcal{V}},[Y, Z]^{\mathcal{V}}\right)-\frac{1}{4} \hat{g}\left([X, Z]^{\mathcal{V}},[Y, W]^{\mathcal{V}}\right) .
\end{aligned}
$$

In our situation, we know the Riemann tensor of $(\mathcal{M}, g)$ and the conformal transformation between $g$ and $\hat{g}$, so we will be able to compute the first term in the right-hand side. For the remaining terms, we need to compute the vertical part of the commutator of two arbitrary horizontal vectors. We have two distributions to consider. In order to deal with both of them in parallel and given their characterization as $\bar{P}_{\alpha}=\epsilon P_{\alpha}, \epsilon= \pm 1$, we introduce the following notation (we work always away from fixed points of $\xi$ ). The distribution $\left\{\mathcal{D}^{\epsilon}\right\}$ is defined as $\operatorname{span}\{k, \ell\}$ if $\epsilon=1$ and $\operatorname{span}\{\xi, \operatorname{grad} Z\}$ if $\epsilon=-1$. The corresponding orthogonal projectors are denoted by $\mathfrak{h}^{\mu}{ }_{\alpha}$ (so that, in the notation above $\mathfrak{h}^{\mu}{ }_{\alpha}=h^{\mu}{ }_{\alpha}$ if $\epsilon=1$ and $\mathfrak{h}^{\mu}{ }_{\alpha}=H_{\alpha}^{\mu}$ if $\left.\epsilon=-1\right)$ and we also write $\hat{\mathfrak{h}}_{\alpha \beta}=\mathfrak{h}^{\mu}{ }_{\alpha} \hat{g}_{\mu \beta}$. Note that

$$
\mathfrak{h}_{\alpha}^{\mu} \mathfrak{h}_{\beta}^{\nu} \mathcal{W}_{\mu \nu}=-\epsilon \sqrt{-\epsilon} W_{\alpha \beta}^{\epsilon}
$$

where, naturally, $W_{\alpha \beta}^{\epsilon}$ is $W_{\alpha \beta}^{+}$when $\epsilon=+1$ and $W_{\alpha \beta}^{-}$when $\epsilon=-1$ and the square root is defined by $\sqrt{-\epsilon}=i$ when $\epsilon=1$ and $\sqrt{-\epsilon}=1$ when $\epsilon=-1$.

We have the following result for the vertical parts of the commutator of horizontal fields.

Lemma 12. Assume both $\bar{P}_{\alpha}=\epsilon P_{\alpha}$ and $\xi \neq 0$ everywhere. With the notation above, let $X, Y$ be any pair of horizontal vector fields of the distribution $\left\{\mathcal{D}^{\epsilon}\right\}$. Then, the vertical part of their commutator is

$$
[X, Y]^{\mathcal{V}}=\sqrt{-\epsilon}(\bar{J}-\epsilon J) X^{\mu} Y^{\nu} W_{\mu \nu}^{\epsilon} \xi
$$


Proof. We first note that for any pair of horizontal vectors $X, Y$ and a vertical vector $t$ we have, using the orthogonality of $t$ and $X, Y$,

$$
g([X, Y], t)=t_{\nu}\left(X^{\mu} \nabla_{\mu} Y^{\nu}-X^{\mu} \nabla_{\mu} Y^{\nu}\right)=-X^{\mu} Y^{\nu} h_{\mu}^{\alpha} h_{\nu}^{\beta}\left(\nabla_{\alpha} t_{\beta}-\nabla_{\beta} t_{\alpha}\right) .
$$

We first prove that $[X, Y]^{\mathcal{V}}$ is proportional to $\xi$. It suffices to show that its scalar product with a vertical, nowhere zero vector orthogonal to $\xi$ vanishes.

For the case $\epsilon=+1$, the condition $\nabla_{\alpha} Z=0$ implies $V_{\alpha}=0$ so that, using the projector (70)

$$
0=V_{\alpha}=h_{\beta}^{\alpha} \nabla_{\alpha} y=\nabla_{\alpha} y-\xi_{\alpha}-\frac{N}{g(\xi, k)} k_{\alpha} .
$$

It follows that $t \stackrel{\text { def }}{=} \xi+\frac{N}{g(\xi, k)} k$ is vertical, nowhere zero, orthogonal to $\xi$ and satisfies $\nabla_{\alpha} t_{\beta}-$ $\nabla_{\beta} t_{\alpha}=0$. Consequently $[X, Y]^{\mathcal{V}}$ is orthogonal to $t$ as required. For the case $\epsilon=-1$ the claim follows immediately with the choice $t=\operatorname{grad} Z$ which is again vertical, nowhere zero, orthogonal to $\xi$ and satisfies $\nabla_{\alpha} t_{\beta}-\nabla_{\beta} t_{\alpha}=0$.

In order to determine the proportionality factor between $[X, Y]^{\mathcal{V}}$ and $\xi$ we compute

$$
\begin{aligned}
g\left([X, Y]^{\mathcal{V}}, \xi\right) & =-X^{\mu} Y^{\nu} \mathfrak{h}^{\alpha}{ }_{\mu} \mathfrak{h}^{\beta}{ }_{\nu}\left(\nabla_{\alpha} \xi_{\beta}-\nabla_{\beta} \xi_{\alpha}\right)= \\
& =-X^{\mu} Y^{\nu} \mathfrak{h}^{\alpha}{ }_{\mu} \mathfrak{h}^{\beta}{ }_{\nu}\left(R \mathcal{W}_{\alpha \beta}+\overline{R \mathcal{W}}_{\alpha \beta}\right)=-\sqrt{-\epsilon}(\bar{R}-\epsilon R) X^{\mu} Y^{\nu} W_{\mu \nu}^{\epsilon}
\end{aligned}
$$

where in the third equality we used (173). The lemma follows from (57) and $g(\xi, \xi)=-N$.

We can now determine the geometry of the quotient in the special cases.

Proposition 4. Assume both $\bar{P}_{\alpha}=\epsilon P_{\alpha}$ and $\xi \neq 0$ everywhere. Choose any point $p \in \mathcal{M}$ and a sufficiently small neighbourhood $U_{p}$ of $p$ where Lemma 5 applies. Then, the curvature scalar of $\left(U_{p} / \sim, \hat{\mathfrak{h}}\right)$ reads

(A) $R(\hat{\mathfrak{h}})=2 \Lambda$.

(B) $R(\hat{\mathfrak{h}})= \begin{cases}2\left(c+2 \Lambda Z^{2}\right) & \text { if } \quad \epsilon=1 \\ 2\left(-c+2 \Lambda y^{2}\right) & \text { if } \quad \epsilon=-1\end{cases}$

Remark. In the case $\epsilon=1$ we can equivalently write $R(\hat{h})=-2 u^{\prime}(Z)$ where $u$ is the real function defined in the Remark after Proposition 2 .

Proof: We have already indicated the steps to prove this result. We start with the determination of $R_{\alpha \beta \mu \nu} \mathfrak{h}^{\alpha}{ }_{\rho} \mathfrak{h}^{\beta}{ }_{\sigma} \mathfrak{h}^{\mu}{ }_{\kappa} \mathfrak{h}^{\nu}{ }_{\delta}$. From (22) and (42) and recalling (73),

$$
\mathcal{C}_{\alpha \beta \mu \nu} \mathfrak{h}_{\rho}^{\alpha} \mathfrak{h}^{\beta}{ }_{\sigma} \mathfrak{h}^{\mu}{ }_{\kappa} \mathfrak{h}^{\nu}{ }_{\delta}=(3 J R-\Lambda)\left(-\epsilon W_{\rho \sigma}^{\epsilon} W_{\kappa \delta}^{\epsilon}+\frac{1}{3}\left(\mathfrak{h}_{\rho \kappa} \mathfrak{h}_{\sigma \delta}-\mathfrak{h}_{\rho \delta} \mathfrak{h}_{\sigma \kappa}\right)\right)
$$

Since $W_{\rho \sigma}^{\epsilon} W_{\kappa \delta}^{\epsilon}$ has the same symmetries as a Riemann tensor in two-dimensions and $W_{\alpha \beta}^{\epsilon} W^{\epsilon \alpha \beta}=$ $-\epsilon$ it follows

$$
\epsilon W_{\rho \sigma}^{\epsilon} W_{\kappa \delta}^{\epsilon}=\mathfrak{h}_{\rho \kappa} \mathfrak{h}_{\sigma \delta}-\mathfrak{h}_{\rho \delta} \mathfrak{h}_{\sigma \kappa}
$$


Taking the real part in (74) and using (13) it follows

$$
R_{\alpha \beta \mu \nu} \mathfrak{h}^{\alpha}{ }_{\rho} \mathfrak{h}^{\beta}{ }_{\sigma} \mathfrak{h}^{\mu}{ }_{\kappa} \mathfrak{h}^{\nu}{ }_{\delta}=(\Lambda-J R-\bar{J} \bar{R})\left(\mathfrak{h}_{\rho \kappa} \mathfrak{h}_{\sigma \delta}-\mathfrak{h}_{\rho \delta} \mathfrak{h}_{\sigma \kappa}\right)
$$

The next step is to perform the conformal rescaling $\hat{g}=\Omega^{-1} g$ and obtain the corresponding Riemann tensor. The distribution $\left\{\mathcal{D}^{\epsilon}\right\}$ is such that (recall expression (35) for $t_{\mu \alpha}$ )

$$
\mathfrak{h}^{\mu}{ }_{\nu} P_{\mu}=0, \quad \mathfrak{h}^{\mu}{ }_{\nu} \mathfrak{h}^{\alpha}{ }_{\beta} t_{\mu \alpha}=\frac{\epsilon R \bar{R}}{2} \mathfrak{h}_{\nu \beta}
$$

Thus, equation (46) implies, using (57),

$$
\mathfrak{h}_{\nu}^{\mu} \mathfrak{h}_{\beta}^{\alpha} \nabla_{\mu} P_{\alpha}=-\frac{1}{2} N(J+\epsilon \bar{J}) \mathfrak{h}_{\nu \beta}
$$

Given that $\bar{P}_{\alpha}=\epsilon P_{\alpha}$, equations (66) and (75) yield

$$
\begin{aligned}
& \nabla_{\alpha} \Omega=-\Omega(J+\epsilon \bar{J}) P_{\alpha}, \\
& \mathfrak{h}^{\mu}{ }_{\nu} \mathfrak{h}_{\beta}^{\alpha} \nabla_{\mu} \nabla_{\alpha} \Omega=\frac{1}{2} N \Omega(J+\epsilon \bar{J})^{2} \mathfrak{h}_{\nu \beta} .
\end{aligned}
$$

The standard transformation law for the Riemann tensor under conformal rescaling gives, after a straightforward calculation,

$$
{ }^{\hat{g}} R_{\alpha \beta \mu \nu} \mathfrak{h}^{\alpha}{ }_{\rho} \mathfrak{h}^{\beta}{ }_{\sigma} \mathfrak{h}^{\mu}{ }_{\kappa} \mathfrak{h}^{\nu}{ }_{\delta}=\frac{1}{\Omega}\left(\Lambda+\frac{1}{4} N(J+\epsilon \bar{J})^{2}-J R-\overline{J R}\right)\left(\mathfrak{h}_{\rho \kappa} \mathfrak{h}_{\sigma \delta}-\mathfrak{h}_{\rho \delta} \mathfrak{h}_{\sigma \kappa}\right)
$$

Inserting this into Proposition 3 and using Lemma 12 gives

$$
{ }^{\hat{\mathfrak{h}}} R_{A B C D}=\Omega\left(\Lambda+\frac{N}{4}(J+\epsilon \bar{J})^{2}-J R-\bar{J} \bar{R}+\frac{3 N}{4}(\bar{J}-\epsilon J)^{2}\right)\left(\hat{\mathfrak{h}}_{A C} \hat{\mathfrak{h}}_{B D}-\hat{\mathfrak{h}}_{A D} \hat{\mathfrak{h}}_{B C}\right) .
$$

The Proposition now follows by explicit substitution of the formulae in Lemma 4 in this expression and using (58,59).

\subsection{Spacetime geometry}

We are ready to prove our main results of this section, namely to determine the spacetime metric under our characterization hypotheses. As already discussed, the strategy is to use the information in the previous two subsections concerning the Riemannian submersions and integrate the field equations from the quotient up to the spacetime. We need to distinguish two cases depending on whether or not $\xi$ is orthogonal to $k$ and $\ell$.

Theorem 2. Let $(\mathcal{M}, g)$ be a $\Lambda$-vacuum, non-locally flat, spacetime admitting a Killing vector $\xi$. Let $\mathcal{F}_{\mu \nu}$ be the self-dual Killing form of $\xi$ and assume that $\mathcal{F}^{2} \neq 0$ everywhere on $\mathcal{M}$ and that the self-dual Weyl tensor $\mathcal{C}_{\alpha \beta \mu \nu}$ satisfies (22). Let $k$ and $\ell$ be the two 
eigenvectors of $\mathcal{F}_{\alpha \beta}$ and let $p \in \mathcal{M}$ be such that $\left.\xi\right|_{p} \notin \operatorname{span}\left\{\left.k\right|_{p},\left.\ell\right|_{p}\right\}^{\perp} \subset T_{p} \mathcal{M}$. Then, there exists an open, connected neighbourhood $U_{p}$ of $p$ with $\xi=\partial_{v}$ and such that $g$ takes the form

$$
g=-N(d v-\hat{\boldsymbol{w}})^{2}+2\left(d y-\star_{\hat{h}} d Z\right)(d v-\hat{\boldsymbol{w}})+\Omega \hat{h} .
$$

where $\hat{h}$ is a Riemannian metric on a two-dimensional manifold $S_{p}$ with Gaussian curvature

$$
K(\hat{h})=-u^{\prime} \circ Z,
$$

$\hat{\boldsymbol{w}}$ is a one-form on the metric $\hat{h}$ satisfying the equation

$$
\hat{d} \hat{\boldsymbol{w}}=(f \circ Z) \hat{\eta},
$$

and $Z$ is a function on the metric $\hat{h}$ whose Hessian satisfies

$$
\text { Hess } Z=(u \circ Z) \hat{h} \text {. }
$$

Here, $u^{\prime}$ is the derivative of $u, \hat{\eta}$ and $\star_{\hat{h}}$ denote the volume 2-form and its corresponding Hodge dual of the metric $\hat{h}$ and $\hat{d}$ is the exterior differential on $S_{p}$. The functions $N, \Omega$ : $\mathcal{M} \rightarrow \mathbb{R}$ and $u, f: \mathbb{R} \rightarrow \mathbb{R}$ belong to one of the following two classes

(A) $N=c-\Lambda\left(y^{2}-Z^{2}\right), \quad \Omega=1, \quad u(\zeta)=-\Lambda \zeta, \quad f=0, \quad \Lambda \neq 0$.

(B) $N=c-\frac{\Lambda}{3}\left(y^{2}-Z^{2}\right)-\frac{b_{1} y+b_{2} Z}{y^{2}+Z^{2}}, \quad \Omega=y^{2}+Z^{2}, \quad u(\zeta)=\frac{b_{2}}{2}-c \zeta-\frac{2 \Lambda}{3} \zeta^{3}, \quad f=2 \zeta$. where $b_{1}, b_{2}, c$ are arbitrary real constants.

Proof: Since $\left.\xi\right|_{p}$ is not simultaneously orthogonal to $\left.k\right|_{p}$ and $\left.\ell\right|_{p}$ we can assume, after interchanging $\ell$ and $k$ if necessary, that $g(\xi, k) \neq 0$ at $p$. Choose $U_{p}$ an open, connected neighbourhood of $p$ where $g(\xi, k) \neq 0$ everywhere. Without loss of generality we can assume $g(\xi, k)=1$ on $U_{p}$. From

$$
\xi_{\alpha}[\xi, k]^{\alpha}=\xi_{\alpha}\left(\xi^{\mu} \nabla_{\mu} k^{\alpha}-k^{\mu} \nabla_{\mu} \xi^{\alpha}\right)=\xi^{\mu} \nabla_{\mu} g(\xi, k)=0,
$$

together with $[\xi, k] \propto k$ we conclude $[\xi, k]=0$. Let $\{\mathcal{D}\}$ be the distribution defined in Proposition 2 and restrict $U_{p}$ further, if necessary, so that Lemma 5 can be applied to the spacetime $(\mathcal{M}, \hat{g})$. Denote by $\pi$ the corresponding submersion and $\hat{h}$ the metric of $S_{p} \stackrel{\text { def }}{=} U_{p} / \sim$. We can assume without loss of generality that $S_{p}$ is connected and $U_{p}=I_{1} \times I_{2} \times S_{p}$, where $I_{1}$ and $I_{2}$ are open intervals of the real line. After restricting $S_{p}$ if necessary, let us introduce coordinates $\left\{\hat{x}^{A}\right\}$ in $S_{p}$. As in [31] we introduce a coordinate system in $U_{p}$ as follows. Since the bundle $\left(U_{p}, S_{p}, \pi\right)$ is trivial, select a global section $\hat{\sigma}: S_{p} \longrightarrow U_{p}$. The sets $\Sigma_{y_{0}} \stackrel{\text { def }}{=}\left\{y=y_{0}\right\}$ are smooth hypersurfaces (because $\nabla_{\alpha} y \neq 0$ everywhere) and transversal to the fibers $\pi^{-1}(\hat{x}), \hat{x} \in S_{p}$ (because $k(y) \neq 0$ everywhere). Consider an arbitrary point $s \in U_{p}$ and let $y_{s}=y(s)$. The point $s^{\prime}=\hat{\sigma}(\pi(s))$ belongs to the same fiber as $s$. Consider the integral line of $k$ passing through $s^{\prime}$ and let $r$ be the 
intersection of this line with $\Sigma_{y_{s}}$ (this intersection exists and it is unique, possibly after restricting $U_{p}$ further, because $k$ is everywhere transverse to $\left\{\Sigma_{y}\right\}$ ). The points $s$ and $r$ belong to the same fibre and to the same hypersurface $\Sigma_{y_{s}}$. Since $\xi$ is tangent to the fibers and tangent to $\Sigma_{y_{s}}$, there exists an integral line of $\xi$ connecting $r$ and $s$. Let $v$ be the natural parameter of this curve starting at $r$ (i.e. $\xi(v)=1$ with $v(r)=0$ ) and define $v_{s}=v(s)$. By construction, it follows that the set of values $\left\{v(s), y(s), x^{A} \stackrel{\text { def }}{=} \hat{x}^{A}(\pi(s))\right\}$ define a coordinate system on $U_{p}$. Moreover, $\xi=\partial_{v}$ and $k=\partial_{y}$ in these coordinates (recall that we have chosen $g(\xi, k)=1$ and hence $k(y)=1$ ). Given that $k$ is null, it follows that the one-form $\boldsymbol{k}$ reads

$$
\boldsymbol{k}=d v-\boldsymbol{w}
$$

for some one-form $\boldsymbol{w}$ that satisfies $£_{\xi} \boldsymbol{w}=0$ due to $£_{\xi} \boldsymbol{k}=0$. The last statement in Lemma 7 implies also that $£_{k} \boldsymbol{k}=0$ so that $£_{k} \boldsymbol{w}=0$ too. Thus, there is a one-form $\hat{\boldsymbol{w}}$ on $\left(S_{p}, \hat{h}\right)$ satisfying $\boldsymbol{w}=\pi^{\star} \hat{\boldsymbol{w}}$. The explicit form of the projector (70) and the definition $h_{\beta}^{\alpha} \nabla_{\alpha} y=V_{\beta}$ implies

$$
\xi_{\beta}=\nabla_{\beta} y-N k_{\beta}-V_{\beta}
$$

which, inserted in (70), provides

$$
g_{\alpha \beta}=\Omega \hat{h}_{\alpha \beta}-N k_{\alpha} k_{\beta}+k_{\alpha}\left(\nabla_{\beta} y-V_{\beta}\right)+k_{\beta}\left(\nabla_{\alpha} y-V_{\alpha}\right) .
$$

From Lemma 10 we have $\boldsymbol{V}=\pi_{\star}\left(\star_{\hat{h}} d \hat{Z}\right)$. Since in the coordinates $\left\{v, y, x^{A}\right\}$ the submersion $\pi$ takes a trivial form, it is safe to use the same notation for objects on the quotient and for corresponding objects on the spacetime (this applies in particular to $\hat{h}, \hat{\boldsymbol{w}}$ and to $\hat{Z}$ ). Thus the metric $g$ in (80) takes the form claimed in the Theorem. It only remains to show that the field equations (76), (177) and (78) hold.

From Proposition 2 we have

$$
D_{A} D_{B} Z=(u \circ Z) \hat{h}_{A B}
$$

where as before $D$ denotes the covariant derivative in $\left(S_{p}, \hat{h}\right)$. This proves (178). The Ricci identity applied to this equations implies

$$
R(h) D_{A} \hat{Z}=-2 u^{\prime}(\hat{Z}) D_{A} \hat{Z} .
$$

Since $S_{p}$ is connected, $d \hat{Z}$ is either non-zero on an open dense set or identically zero In the former case we can drop $D_{A} \hat{Z}$ and we conclude (76). The case $d Z=0$ has been dealt with in Proposition 4 (see in particular the Remark after this Proposition) and also leads to (176). Finally, (179) and Lemma 9 imply

$$
\hat{d} \hat{\boldsymbol{w}}=i(\bar{J}-J) \Omega \hat{\boldsymbol{\eta}} .
$$

In case (A) we have $J=0$ so (77) holds with $f=0$ and in case (B) we have $J=-1 / P$ and $\Omega=P \bar{P}$. Hence, $i(\bar{J}-J) \Omega=2 Z$, as claimed.

Having dealt with the case when $\xi$ is not orthogonal to the eigenspace of $\mathcal{F}_{\alpha \beta}$ we study next the remaining case when $\xi$ is orthogonal to both $k$ and $\ell$. 
Theorem 3. Let $(\mathcal{M}, g)$ be a $\Lambda$-vacuum, non-locally flat, spacetime admitting a Killing vector $\xi$ with no fixed points. Let $\mathcal{F}_{\mu \nu}$ be the self-dual Killing form of $\xi$ and assume that $\mathcal{F}^{2} \neq 0$ everywhere on $\mathcal{M}$ and that the self-dual Weyl tensor $\mathcal{C}_{\alpha \beta \mu \nu}$ satisfies (22). Let $k$ and $\ell$ be the two real null eigenvectors of $\mathcal{F}_{\alpha \beta}$ and assume that $g(\xi, k)=g(\xi, \ell)=0$ on $\mathcal{M}$. Then, locally the metric $g$ adopts the form

$$
g=\frac{1}{-N} d Z^{2}-N(d v-\hat{\boldsymbol{w}})^{2}+\Omega \hat{H} .
$$

where $\xi=\partial_{v}, \hat{H}$ is a 2-dimensional Lorentzian metric of constant curvature $\kappa, \hat{\boldsymbol{w}}$ is a one-form on the metric $\hat{H}$ satisfying the equation

$$
\hat{d} \hat{\boldsymbol{w}}=2 y \hat{\boldsymbol{\eta}}
$$

where $y$ is an arbitrary real constant and $\hat{d}$ and $\hat{\boldsymbol{\eta}}$ are the exterior differential and volume form of the metric $\hat{H}$. The functions $N$ and $\Omega$ belong to one of the following two classes:

$$
\text { (B) } \quad N=\frac{1}{y^{2}+Z^{2}}\left(c\left(Z^{2}-y^{2}\right)-b_{2} Z+\frac{\Lambda}{3}\left(Z^{4}+3 y^{4}\right)\right), \quad \Omega=y^{2}+Z^{2}, \quad \kappa=2 \Lambda y^{2}-c \text {. }
$$

where $b_{2}, c$ are arbitrary (real) constants.

Proof: Let $p \in \mathcal{M}$ be any point and choose $U_{p}$ an open, connected neighbourhood so that Lemma 5 can be applied to the distribution $\left\{\mathcal{D}^{-}\right\}$and with respect to the metric $(\mathcal{M}, \hat{g})$. Denote by $\pi$ the corresponding submersion and $\hat{H}$ the (Lorentzian) metric of $S_{p} \stackrel{\text { def }}{=} U_{p} / \sim$. Proposition 4 shows that $\hat{H}$ is a two-dimensional metric of constant curvature $\kappa$ as given in cases (A) and (B) of the Theorem. Let $\hat{\boldsymbol{\eta}}$ be a volume form of $\left(S_{p}, \hat{H}\right)$ (the orientation will be chosen later) and define $\hat{\eta}_{\alpha \beta}=\pi^{\star}(\hat{\boldsymbol{\eta}})_{\alpha \beta}$. Since $W_{\alpha \beta}^{-}$is a horizontal two-form, it must be proportional to $\hat{\eta}_{\alpha \beta}$. Its square norm in the metric $\hat{g}$ is $-2 \Omega^{2}$ (cf. (37)) which forces $W_{\alpha \beta}^{-}= \pm \Omega \hat{\eta}_{\alpha \beta}$. Choose the orientation in $\left(S_{p}, \hat{H}\right)$ so that the plus sign holds.

In order to construct the metric, the starting point is (172).$\nabla_{\alpha} Z$ is nowhere zero (because $\xi$ has no fixed point in $\mathcal{M}$ ), so $Z$ can be used as a coordinate on $U_{p}$ and we can write

$$
g=-\frac{1}{N}\left(\boldsymbol{\xi} \otimes \boldsymbol{\xi}+d Z^{2}\right)+\Omega \hat{H} .
$$

It only remains to find $\boldsymbol{\xi}$. From $d \boldsymbol{\xi}=\mathcal{F}+\overline{\mathcal{F}}=R \mathcal{W}+\overline{R \mathcal{W}}$ and $\nabla_{\alpha} N=R P_{\alpha}+\overline{R P}_{\alpha}$ (recall that $2 N=(\chi+\bar{\chi})$ and $\left.\nabla_{\alpha} \chi=2 R P_{\alpha}\right)$ the following general identity follows

$$
d\left(N^{-1} \boldsymbol{\xi}\right)=N^{-1}\left(R \mathcal{W}+\overline{R \mathcal{W}}+N^{-1} \boldsymbol{\xi} \wedge(R \boldsymbol{P}+\overline{R \boldsymbol{P}})\right) .
$$

In the present case $\overline{\boldsymbol{P}}=-\boldsymbol{P}$ and (61) holds, so that

$$
d\left(N^{-1} \boldsymbol{\xi}\right)=N^{-1}(R+\bar{R}) \boldsymbol{W}^{-}=(J+\bar{J}) \Omega \hat{\boldsymbol{\eta}},
$$


where in the second equality we used (57) and the relationship between $\boldsymbol{W}^{-}$and $\hat{\boldsymbol{\eta}}$ just discussed. In either case $(A)$ or $(B)$ we have $(J+\bar{J}) \Omega=-2 y$. Let $\hat{\boldsymbol{w}}$ be a one-form on $\left(S_{p}, \hat{H}\right)$ satisfying

$$
\hat{d} \hat{\boldsymbol{w}}=2 y \hat{\boldsymbol{\eta}}
$$

It follows from (82) that $d\left(N^{-1} \boldsymbol{\xi}+\pi^{\star}(\hat{\boldsymbol{w}})\right)=0$, and consequently, the existence (restricting $U_{p}$ further if necessary) of a function $v: U_{p} \rightarrow \mathbb{R}$ such that

$$
\boldsymbol{\xi}=N\left(d v-\pi^{\star}(\hat{\boldsymbol{w}})\right)
$$

With the slight abuse of notation of naming $\pi^{\star}(\hat{\boldsymbol{w}})$ still as $\hat{\boldsymbol{w}}$, the metric given in the Theorem follows. Finally, expressions (81) are a consequence of the general formulae in Lemma 4 after using (58)-(59) with $\epsilon=-1$.

\section{$5 \quad$ Semiglobal considerations}

So far we have found the local form of the metric under the assumption $\mathcal{F}^{2} \neq 0$ everywhere and we have split the analysis depending on whether $\xi \notin \operatorname{span}\{k, \ell\}^{\perp}$ everywhere or $\xi \in$ $\operatorname{span}\{k, \ell\}^{\perp}$ everywhere. In a given spacetime it may happen a priori that $\mathcal{F}^{2}$ becomes zero and/or that those different situations can occur in disjoint open sets. We analyze in this section whether this is possible or not.

Throughout this section we assume that $(\mathcal{M}, g)$ is a smooth $\Lambda$-vacuum spacetime admitting a Killing vector $\xi$ such that (22) holds with a smooth proportionality function $Q$. Note that smoothness of $Q$ is an assumption, because in principle it may be the case that $Q$ diverges somewhere, while $\mathcal{F}_{\alpha \beta} \rightarrow 0$ at the same place so that the right-hand side in (22) stays smooth. However, this is not what is meant when saying that $\mathcal{C}_{\alpha \beta \mu \nu}$ and $\mathcal{F}_{\alpha \beta} \mathcal{F}_{\mu \nu}-\frac{1}{3} \mathcal{F}^{2} \mathcal{I}_{\alpha \beta \mu \nu}$ are proportional everywhere. We have the following result concerning $\mathcal{F}^{2}$.

Proposition 5. Define $\mathcal{M}_{\mathcal{F}^{2}} \stackrel{\text { def }}{=}\left\{p \in \mathcal{M} ;\left.\mathcal{F}^{2}\right|_{p} \neq 0\right\}$ and assume $\mathcal{M}_{\mathcal{F}^{2}} \neq \emptyset$ and that there is $p \in \mathcal{M}_{\mathcal{F}^{2}}$ with $Q(p) \neq 0$. Then $\mathcal{M}_{\mathcal{F}^{2}}=\mathcal{M}$.

Remark: If $Q=0$ everywhere on $\mathcal{M}_{\mathcal{F}^{2}}$, then the spacetime on this open set is locally isometric to the Minkowski $(\Lambda=0)$, de Sitter $(\Lambda>0)$ or Anti-de Sitter $(\Lambda<0)$ spacetimes. In the case $\Lambda \neq 0$, these spacetimes admit Killing vectors for which $\mathcal{F}^{2}$ is not identically zero and vanishes somewhere (an example is the Killing vector $\xi=y\left(\partial_{t}+\partial_{x}\right)+(t-x) \partial_{y}$ in the de Sitter space in conformally flat coordinates $\{t, x, y, z\}$, for which $\mathcal{F}^{2} \propto(t-x)^{2}$ with a nowhere zero proportionality factor). Thus, the condition on the existence of $p$ is necessary when $\Lambda \neq 0$. On the other hand, if $\Lambda=0$, then the spacetime on $\mathcal{M}_{\mathcal{F}^{2}}$ would be locally Minkowski for which $\mathcal{F}^{2}$ is constant for any Killing vector. So automatically we would have $\mathcal{M}_{\mathcal{F}^{2}}=\mathcal{M}$ in this case. This shows that the condition on the existence of $p$ can be dropped in the $\Lambda=0$ case. 
Proof: Consider the connected component $\mathcal{M}_{\mathcal{F}^{2}}^{0}$ of $\mathcal{M}_{\mathcal{F}^{2}}$ containing $p$ and assume $\mathcal{M}_{\mathcal{F}^{2}}^{0} \neq \mathcal{M}_{\mathcal{F}^{2}}$. Let $q \in \partial \mathcal{M}_{\mathcal{F}^{2}}^{0}$ and consider the smooth function $Q \mathcal{F}^{2}$ on $\mathcal{M}$, which on $\mathcal{M}_{\mathcal{F}^{2}}^{0}$ takes the form

$$
-\frac{1}{4} Q \mathcal{F}^{2}=R^{2} Q=3 J R-\Lambda .
$$

The left-hand side approaches zero when we approach $q$. We know that $J$ is either identically zero or nowhere zero on $\mathcal{M}_{\mathcal{F}^{2}}^{0}$. In the former case, the right hand-side of (83) takes the constant value $-\Lambda$ which cannot approach 0 at $q$ unless $\Lambda=0$, but then $Q$ vanishes identically on $\mathcal{M}_{\mathcal{F}^{2}}^{0}$ against hypothesis.

For the remaining case $J \neq 0$ on $\mathcal{M}_{\mathcal{F}^{2}}^{0}$, we use the expressions in Lemma 4 to compute the right-hand side of (83) as

$$
R^{2} Q=\left.(3 J R-\Lambda)\right|_{\mathcal{M}_{\mathcal{F}^{2}}^{0}}=-\frac{3 b}{2 P^{3}}
$$

Since $Q(p) \neq 0$, the constant $b$ cannot be zero. Hence, it must be $P \rightarrow \infty$ when we approach $q$. Since $R=b /\left(2 P^{2}\right)-\Lambda P / 3$ and $R \rightarrow 0$ at $q$, we necessarily have $\Lambda=0$. But then $Q=\frac{3 J}{R}=\frac{-6 P}{b}$ which diverges at $q$ against hypothesis.

Concerning the possibility that the functions $g(\xi, k)$ and $g(\xi, \ell)$ vanish at a point $p \in \mathcal{M}$, we can prove the following general result concerning the special cases of subsection 3.1.

Proposition 6. Assume that $\left.\boldsymbol{\xi} \wedge \boldsymbol{q}\right|_{p}=0$ at $p \in \mathcal{M}$. Then, there exists $\epsilon \in\{-1,1\}$ such that either

1. $q=\epsilon \xi$ everywhere, or

2. $p$ belongs to a 2-dimensional connected and totally geodesic surface $\mathcal{B}$, such that $\left.q\right|_{\mathcal{B}}=$ $\left.\epsilon \xi\right|_{\mathcal{B}}$ on all $\mathcal{B}$,

depending on whether $R-\epsilon \bar{R}-N(J-\epsilon \bar{J})$ vanishes (case 1) or not (case 2) at $p$. In the latter case, $\mathcal{B}$ is spacelike or timelike if $\epsilon$ is -1 or 1 , respectively.

Remark: At $p$ one has, from the results of subsection 3.1, that $\left.\boldsymbol{P}\right|_{p}=\left.\epsilon \overline{\boldsymbol{P}}\right|_{p}$ (allowing also the case when they vanish identically), ergo $\left.d y\right|_{p}=0$ (if $\epsilon=-1$ ) or $\left.d Z\right|_{p}=0$ (if $\epsilon=1$ ) From (44), we have that

$$
\left.(R-\epsilon \bar{R}-N(J-\epsilon \bar{J}))\right|_{p}=\left\{\begin{array}{cl}
\left.\nabla_{\mu} \nabla^{\mu} y\right|_{p} & \text { if } \epsilon=-1 \\
\left.i \nabla_{\mu} \nabla^{\mu} Z\right|_{p} & \text { if } \epsilon=1
\end{array}\right.
$$

so one can reformulate the proposition in terms of the vanishing or not of the differential and the D'Alembertian of $y(\epsilon=-1)$ or $Z(\epsilon=1)$ at $p$.

Proof: From the results in Subsection 3.1 we know that $\left.\boldsymbol{\xi} \wedge \boldsymbol{q}\right|_{p}=0$ implies that there is $\epsilon \in\{-1,1\}$ such that $\left.q\right|_{p}=\left.\epsilon \xi\right|_{p}$ (if $\left.\xi\right|_{p}=0$ then $\left.q\right|_{p}=0$ and the two choices of $\epsilon$ are 
allowed). From Proposition 1 there is a Killing vector $\varsigma$ that vanishes at $p$, given by the constants $C \neq 0$ and $A=-\epsilon C$ in case (A), and $A=C\left(\left.P\right|_{p}-\left.\epsilon \bar{P}\right|_{p}\right)^{2}$ in case (B). Thus $p$ is a fixed point of this Killing $\varsigma$. The connected set of fixed points of $\varsigma$ containing $p$ is a totally geodesic, smooth surface with the same dimension and causal character as the vector subspace $\mathcal{V}_{p}(\varsigma)=\left\{u \in T_{p} \mathcal{M} ;\left.d \varsigma\right|_{p}(u, \cdot)=0\right\}$ (see e.g. Lemma 5 in [34]). So, we must compute the Killing 2-form $\nabla_{\mu} \varsigma_{\nu}$ at $p$. By using formulas (477) and $\nabla_{\mu} P=P_{\mu}=\epsilon \bar{P}_{\mu}$ and recalling (61) and (63), a somewhat long calculation leads to

$$
\left.\nabla_{\mu} \varsigma_{\nu}\right|_{p}=\left.\left.\sqrt{-\epsilon} G\right|_{p}(R-\epsilon \bar{R}-N(J-\epsilon \bar{J})) W_{\mu \nu}^{\epsilon}\right|_{p}
$$

where $G$ is as in the proof of Proposition 1. Thus, if $R-\epsilon \bar{R}-N(J-\epsilon \bar{J})$ vanishes at $p$ the Killing vector $\varsigma$ vanishes everywhere on $\mathcal{M}$ and we have (using again the notation in the proof of Prop. 1) $q=-G^{-1} F \xi$ everywhere with $\left.G^{-1} F\right|_{p}=-\epsilon$. The function $-G^{-1} F$ is smooth in $\mathcal{M}$ and according to Subsect. 3.1 it must be constant and equal to $\epsilon$ and we are in case 1 of the Proposition.

If on the other hand

$$
\left.(R-\epsilon \bar{R}-N(J-\epsilon \bar{J}))\right|_{p} \neq 0
$$

then the vector subspace $\mathcal{V}_{p}(\varsigma)$ is two-dimensional and spacelike if $\epsilon=-1$ or timelike if $\epsilon=1$. To finish the proof, observe that the Killing vector $\varsigma$ vanishes on all of $\mathcal{B}$, and therefore $\left.\boldsymbol{\xi} \wedge \boldsymbol{q}\right|_{\mathcal{B}}=0$. Invoking again Subsection 3.1 we know that this can only happen if, at each point of $\mathcal{B}$, either $q= \pm \xi$ or $q=0=\xi$. If $\left.\xi\right|_{\mathcal{B}} \neq 0$ everywhere then $q_{\mathcal{B}}=\left.\epsilon \xi\right|_{\mathcal{B}}$ on all $\mathcal{B}$. If on the other hand there is a $p^{\prime} \in \mathcal{B}$ such that $\left.\xi\right|_{p^{\prime}}=0$ (and thus $\left.q\right|_{p^{\prime}}=0$ too), the condition (84) may hold or not at $p^{\prime}$. If it does not for either value of $\epsilon$ then the same reasoning as above, applied now to the point $p^{\prime}$, proves that $q=\epsilon \xi$ everywhere for that choice of $\epsilon$. Otherwise, (84) at $p^{\prime}$-where $N=0$ - provides $\left.R\right|_{p^{\prime}}-\left.\epsilon \bar{R}\right|_{p^{\prime}} \neq 0$ for both choices of $\epsilon$, so that $R$ can be neither real nor purely imaginary at $p^{\prime}$. But then $p^{\prime}$ is a fixed point of $\xi$ whose Killing 2 -form is given by (48), and thus $\operatorname{dim} \mathcal{V}_{p}^{\prime}(\xi)=0$, so that $p^{\prime} \in \mathcal{B}$ is isolated and $\xi$ does not vanish around $p^{\prime}$ on $\mathcal{B}$. Thus, again there is an $\epsilon \in\{-1,1\}$ such that $\left.q\right|_{\mathcal{B}}=\left.\epsilon \xi\right|_{\mathcal{B}}$.

From item 2 in the Proposition and (56) we have, on $\mathcal{B}, \overline{\boldsymbol{P}}=\epsilon \boldsymbol{P}$. For $\epsilon=1$ this means $\left.d Z\right|_{\mathcal{B}}=0$ and for $\epsilon=-1,\left.d y\right|_{\mathcal{B}}=0$. Given that $\mathcal{B}$ is connected it follows

$$
\left.y\right|_{\mathcal{B}}=y_{p} \text { if } \epsilon=-1 ;\left.\quad Z\right|_{\mathcal{B}}=Z_{p} \text { if } \epsilon=1 .
$$

As an immediate consequence of the previous Proposition we have

Corollary 1. Assume that $\left.g(\xi, k)\right|_{p}=\left.g(\xi, \ell)\right|_{p}=0$ at $p \in \mathcal{M}$. Then, either

1. $g(\xi, k)=g(\xi, \ell)=0$ everywhere on $\mathcal{M}$, or

2. $p$ belongs to a 2-dimensional connected and totally geodesic spacelike surface $\mathcal{B}$ such that $g(\xi, k)$ and $g(\xi, \ell)$ vanish on all $\mathcal{B}$,

depending on whether $R+\bar{R}-N(J+\bar{J})$ vanishes (case 1) or not (case 2) at $p$. 


\section{Alternative forms of the metrics and characteriza- tion results}

The main theorems in the previous sections involve field equations on two dimensional Riemannian manifolds. The following Lemma solves them.

Lemma 13. Let $(S, \hat{h})$ be a two-dimensional oriented Riemannian manifold with smooth metric $\hat{h}$ and volume form $\hat{\eta}$. Let $Z$ be a scalar and $\hat{\boldsymbol{w}}$ a one-form on $S$ satisfying

$$
\text { Hess } Z=(u \circ Z) \hat{h}, \quad \hat{d} \hat{\boldsymbol{w}}=(f \circ Z) \hat{\eta} .
$$

where $u, f: I \subset \mathbb{R} \longrightarrow \mathbb{R}$ are smooth real functions. Then, on the open set $\left\{p \in S ;\left.d Z\right|_{p} \neq\right.$ $0\}$ (if non-empty) there exist local coordinates $\{Z, x\}$ such that

$$
\hat{h}=\frac{d Z^{2}}{V(Z)}+V(Z) d x^{2}, \quad \hat{\boldsymbol{w}}=F(Z) d x+\hat{\boldsymbol{w}}_{0},
$$

where $\frac{d V}{d Z}=2 u(Z), \frac{d F}{d Z}=f(Z)$ and $\hat{\boldsymbol{w}}_{0}$ is a closed one-form. Moreover $\star_{\hat{h}} d Z=-V d x$.

Proof: $\quad$ From $D_{A} D_{B} Z=u(Z) \hat{h}_{A B}$ it follows $D_{A}\left((d Z, d Z)_{\hat{h}}\right)=2 u(Z) D_{A} Z$. On any connected component $U^{0}$ of $\left\{p \in S ;\left.d Z\right|_{p} \neq 0\right\}$ this implies the existence of a function $V: I \longrightarrow \mathbb{R}$ satisfying $V^{\prime}=2 u$ and $(d Z, d Z)=V \circ Z$ (observe that this is positive). The Hodge dual $\star_{\hat{h}} d Z$ is nowhere zero on $U^{0}$ and hence defines a distribution, which is always integrable in two dimensions. Locally, there exists a function $x: U^{0} \longrightarrow \mathbb{R}$ such that $\star_{\hat{h}} d Z=-V(Z) H d x$, where $H(Z, x)$ is a positive function to be determined (the sign is chosen so that $\{d Z, d x\}$ is positively oriented). Given that $\left(\star_{\hat{h}} d Z, \star_{\hat{h}} d Z\right)_{\hat{h}}=(d Z, d Z)_{\hat{h}}=$ $V(Z)$, and $\star_{\hat{h}} d Z$ is orthogonal to $d Z$, the metric on $U^{0}$ takes the local form

$$
\hat{h}=\frac{d Z^{2}}{V(Z)}+V(Z) H^{2} d x^{2}
$$

The ' $x x^{\prime}$ component of (Hess $\left.Z\right)_{A B}=u(Z) \hat{h}_{A B}$ reads $\partial_{Z} H=0$. Redefining $x$ as a function of itself, we can set $H=1$ without loss of generality. This gives the metric in (86). The volume form is $\hat{\boldsymbol{\eta}}=d Z \wedge d x$, so equation (85) is simply $\hat{d} \hat{\boldsymbol{w}}=f(Z) d Z \wedge d x$. With $F(\zeta)$ being any integral of $f(\zeta)$, the general solution of this equation is as in (866). The last statement is obvious from the previous considerations.

The following theorem summarizes the main results so far in a self-contained form.

Theorem 4. Let $(\mathcal{M}, g)$ be a $\Lambda$-vacuum spacetime admitting a Killing vector $\xi$ and corresponding self-dual two-form $\mathcal{F}_{\alpha \beta}$. Assume

$$
\mathcal{C}_{\alpha \beta \mu \nu}=Q\left(\mathcal{F}_{\alpha \beta} \mathcal{F}_{\mu \nu}-\frac{1}{3} \mathcal{F}^{2} \mathcal{I}_{\alpha \beta \mu \nu}\right)
$$


for a smooth function $Q: \mathcal{M} \rightarrow \mathbb{C}$ and that $\exists p \in \mathcal{M}$ such that $\left.Q\right|_{p} \neq 0$ and $\left.\mathcal{F}^{2}\right|_{p} \neq 0$. Then $\mathcal{F}^{2} \neq 0$ everywhere and

$$
q_{\mu} \stackrel{\text { def }}{=} 4\left(\mathcal{F}^{2} \overline{\mathcal{F}}^{2}\right)^{-1 / 2} \xi^{\alpha} \overline{\mathcal{F}}_{\alpha}^{\beta} \mathcal{F}_{\beta \mu}
$$

exists globally.

(A) If $Q \mathcal{F}^{2}-4 \Lambda=0$ at one point, then it vanishes everywhere and $q$ is a Killing vector field satisfying $[\xi, q]=0$. The metric is locally reducible $g=h_{-}+h_{+}$where $h_{-}$ (resp. $h_{+}$) is a two-dimensional Lorentzian (resp. Riemannian) metric of constant curvature $\Lambda$ and $\xi$ is any of the Killing vectors of $h_{-}$or $h_{+}$as long as it satisfies $d \boldsymbol{\xi} \neq 0$ everywhere.

(B) If $Q \mathcal{F}^{2}-4 \Lambda \neq 0$ at one point then it does not vanish anywhere on $\mathcal{M}$ and $\chi_{\alpha} \stackrel{\text { def }}{=} 2 \xi^{\beta} \mathcal{F}_{\beta \alpha}$ is exact $\chi_{\alpha}=\nabla_{\alpha} \tilde{\chi}$ with

$$
\tilde{\chi}=6 \mathcal{F}^{2} \frac{Q \mathcal{F}^{2}+2 \Lambda}{\left(Q \mathcal{F}^{2}-4 \Lambda\right)^{2}} .
$$

Moreover $\exists b_{1}, b_{2}, c \in \mathbb{R}$ such that

$$
36 Q\left(\mathcal{F}^{2}\right)^{\frac{5}{2}}=\left(-b_{2}+i b_{1}\right)\left(Q \mathcal{F}^{2}-4 \Lambda\right)^{3}
$$

and $N-\operatorname{Re}(\tilde{\chi})=c$, where $N \stackrel{\text { def }}{=}-g(\xi, \xi)$ and the vector field

$$
\varsigma^{\mu}=\frac{4}{\left|Q \mathcal{F}^{2}-4 \Lambda\right|^{2}} \xi^{\sigma} \overline{\mathcal{F}}_{\sigma}{ }^{\rho} \mathcal{F}_{\rho}{ }^{\mu}+\operatorname{Re}\left(\frac{\mathcal{F}^{2}}{\left(Q \mathcal{F}^{2}-4 \Lambda\right)^{2}}\right) \xi^{\mu}
$$

is Killing and commutes with $\xi$. Let $\mathcal{L}=\operatorname{span}\{\xi, \varsigma\}$.

(B.i) If $\operatorname{dim}(\mathcal{L})=2$, then $\exists k \in \mathbb{R}$ such that, away from totally geodesic, codimensiontwo, non-degenerate surfaces where a Killing vector in $\mathcal{L}$ vanishes, the metric is, locally

$$
\begin{gathered}
d s^{2}=-N\left(d v-Z^{2} d x\right)^{2}+2(d y+V d x)\left(d v-Z^{2} d x\right)+\left(y^{2}+Z^{2}\right)\left(\frac{d Z^{2}}{V}+V d x^{2}\right) \\
\xi=\partial_{v}, \quad N=c-\frac{\Lambda}{3}\left(y^{2}-Z^{2}\right)-\frac{b_{1} y+b_{2} Z}{y^{2}+Z^{2}}, \quad V=k+b_{2} Z-c Z^{2}-\frac{\Lambda}{3} Z^{4} .
\end{gathered}
$$

(B.ii) If $\operatorname{dim}(\mathcal{L})=1$, then $\exists \epsilon \in\{-1,1\}$ and $\kappa, \beta, n \in \mathbb{R}$ such that, away from fixed points of $\xi$, the metric is, locally,

$$
\begin{aligned}
d s^{2} & =\left\{\begin{array}{lr}
-V(d v-\hat{\boldsymbol{w}})^{2}+2 d x(d v-\hat{\boldsymbol{w}})+\left(\beta^{2}+x^{2}\right) h_{+} & \text {if } \epsilon=1 \\
V^{-1} d x^{2}+V(d v-\hat{\boldsymbol{w}})^{2}+\left(\beta^{2}+x^{2}\right) h_{-} & \text {if } \epsilon=-1
\end{array}\right. \\
V & =\left(\beta^{2}+x^{2}\right)^{-1}\left(-\frac{\Lambda}{3}\left(x^{4}+6 \beta^{2} x^{2}-3 \beta^{4}\right)-\kappa\left(\beta^{2}-x^{2}\right)+n x\right), \\
\xi & =\partial_{v}, \quad \hat{d} \hat{\boldsymbol{w}}=2 \beta \boldsymbol{\eta}_{\boldsymbol{\epsilon}}
\end{aligned}
$$

where $h_{\epsilon}$ is a metric of constant curvature $\kappa$, signature $\{\epsilon, 1\}$ and volume form $\eta_{\epsilon}$ 
Concerning the result in case (A), note that any Killing vector on the sphere satisfies $d \boldsymbol{\xi} \neq 0$ everywhere, but this is not the case for the rest of two-dimensional spaces of constant curvature (Riemannian or Lorentzian), so the condition $d \boldsymbol{\xi} \neq 0$ is a restriction for the choice of Killing in those cases. Nevertheless, in any 2-dimensional space of constant curvature there is an open subset of the Killing algebra satisfying $d \boldsymbol{\xi} \neq 0$ everywhere.

Proof: From (42) we note that $Q \mathcal{F}^{2}-4 \Lambda=0$ is equivalent to $J=0$. Since $J=0$ at one point if and only if $J=0$ everywhere, the first claim follows. So we are in case (A) (in the notation above). From Proposition 1 we have that $q$ is a Killing vector which commutes with $\xi$, hence the Lie algebra they generate has dimension one or two. The former case corresponds, by definition, to the so-called special cases with $J=0$. So either we are in case (A) of Theorem 2 with $Z=0$ (because of (58) with $\epsilon=1$ ) or in case (A) of Theorem 3. The one-form $\hat{\boldsymbol{w}}$ is closed, hence locally exact and a redefinition of $v$ and $x$, respectively, makes it identically zero. Define $h_{-}=-N(y) d v^{2}+2 d y d v, h_{+}=\hat{h}$ in the case $\xi+q=0$ and $h_{-}=\hat{H}, h_{+}=N^{-1}(Z) d Z^{2}+N(Z) d x^{2}$ in the case $\xi-q=0$, where $N(\zeta) \stackrel{\text { def }}{=} c-\Lambda \zeta^{2}$. It is immediate to check that, in all cases, $h_{-}$and $h_{+}$are of constant curvature $\Lambda$.

In the other case — when the Lie algebra is two-dimensional-, from Theorem 2, Proposition 6 and Lemma $13 \exists c, k \in \mathbb{R}$ such that, away from a collection of totally geodesic, nowhere null, codimension-two surfaces where a Killing vector in $\operatorname{span}\{\xi, q\}$ vanishes, the metric is, locally,

$$
d s^{2}=-N d v^{2}+2(d y+V d x) d v+\frac{d Z^{2}}{V}+V d x^{2}
$$

where $\xi=\partial_{v}, N=c-\Lambda\left(y^{2}-Z^{2}\right)$ and $V=k-\Lambda Z^{2}$. Performing the trivial change $d \tilde{x}=d(x+v)$ this can be rewritten as

$$
d s^{2}=-\tilde{N}(y) d v^{2}+2 d y d v+\frac{d Z^{2}}{V}+V d \tilde{x}^{2}
$$

where now $\tilde{N}(y)=N+V=c+k-\Lambda y^{2}$ and the claim follows again.

To conclude the first part of the theorem, it remains to show that (87) holds for any Killing vector $\xi$ of $h_{ \pm}$for which $d \boldsymbol{\xi}$ is nowhere zero. Let $\boldsymbol{\eta}_{ \pm}$be the volume form of $h_{ \pm}$, so we have $d \boldsymbol{\xi}=2 S \boldsymbol{\eta}_{ \pm}$with $S$ nowhere zero. The curvature tensor of a product manifold inherits a product structure. As a consequence, it turns out that the tensor relation (87) contains just one independent equation, which can be computed to be $Q S^{2}= \pm \Lambda$ showing the proportionality claimed for such $\xi$.

Concerning the case $Q \mathcal{F}^{2}-4 \Lambda \neq 0$, by computing $\tilde{\chi}$ as defined in the theorem and using Lemma 4 it follows $\tilde{\chi}=\chi-c$, from which the statement $N-\operatorname{Re}(\tilde{\chi})=c$ follows immediately. The expression involving $-b_{2}+i b_{1}=i b$ also follows by direct computation from Lemma 4. The Killing vector is simply $\varsigma$ in case (B) of Proposition 1 with $A=0$ and $C=1 / 36$, after rewriting $P$ in terms of $\mathcal{F}^{2}$ as

$$
P=\frac{6 i \sqrt{\mathcal{F}^{2}}}{Q \mathcal{F}^{2}-4 \Lambda} .
$$


The form of the metric in case (B.i) is a direct consequence of Theorem 2, Proposition 6] and Lemma 13. Regarding case (B.ii), this is achieved from case (B) in Theorem 3 (for $\epsilon=-1$ ) and from case (B) with $Z=$ const. in Theorem 2 (for $\epsilon=1$; recall that then (59) implies $\left.b_{2}=2 c Z+4 \Lambda Z^{3} / 3\right)$ together with the name substitutions $\{y \rightarrow \beta, Z \rightarrow x, c \rightarrow$ $\left.2 \Lambda \beta^{2}-\kappa, b_{2} \rightarrow n, N \rightarrow-V\right\}$ in the case $\epsilon=-1$ and $\left\{Z \rightarrow \beta, y \rightarrow x, c \rightarrow-2 \Lambda \beta^{2}+\kappa, b_{1} \rightarrow\right.$ $-n, N \rightarrow V\}$ in the case $\epsilon=1$.

We can proceed to the identification of the metrics and consequently obtain characterizations thereof.

Theorem 5. 1. The metric in case (A) corresponds to the uncharged Bertotti-Robinson metric, and in particular it is the Nariai metric for $\Lambda>0$.

2. At points where $\nabla_{\alpha} y \nabla^{\alpha} y \neq 0$, the metric in case (B.i) in Theorem 4 is locally isometric to the uncharged Plebanski metric.

3. At points where the Killing vector $\xi$ is not null the metric in case (B.ii) with $\epsilon=1$ is locally isometric to a spacetime determined by Cahen and Defrise [5] having a 4-dimensional group of isometries acting on timelike (respectively spacelike) hypersurfaces on the regions where $\xi$ is spacelike (resp. timelike). In particular, for $\kappa>0$ they are locally isometric to the Taub-NUT-(A)de Sitter spacetime.

4. The metric in case (B.ii) with $\epsilon=-1$ are the $\Lambda$-vacuum type-D solutions of Kundt's class. They happen to have both principal null directions expansion-and twist-free, and a 4-dimensional group of isometries acting on spacelike hypersurfaces.

Proof: Point 1 follows directly because the generalized Bertotti-Robinson is the general metric product of two 2-dimensional metric of constant curvature, and due to the fact that Nariai's solution corresponds to the case with a positive curvature Riemannian part.

To prove point 2 , in the (B.i) metric we easily find $\nabla_{\alpha} y \nabla^{\alpha} y=\left(y^{2}+Z^{2}\right) W(y)$ with

$$
W(y)=k-b_{1} y+c y^{2}-\frac{\Lambda}{3} y^{4} .
$$

On any domain where $\nabla_{\alpha} y \nabla^{\alpha} y \neq 0$ we can define new coordinates $\tau$ and $\sigma$ by the coordinate change

$$
d \tau=d v-\frac{y^{2}}{W(y)} d y, \quad d \sigma=d x+\frac{1}{W(y)} d y
$$

After elementary manipulations the line-element in (B.i) becomes

$d s^{2}=\frac{1}{y^{2}+Z^{2}}\left(V(Z)\left(d \tau+y^{2} d \sigma\right)^{2}-W(y)\left(d \tau-Z^{2} d \sigma\right)^{2}\right)+\left(y^{2}+Z^{2}\right)\left(\frac{d Z^{2}}{V(Z)}+\frac{d y^{2}}{W(y)}\right)$.

which is exactly the form of the Plebanski metric as given in formula (21.16) in [45] with the electric and magnetic charges set to zero. 
Similarly, to prove point 3 , on any domain where $g(\xi, \xi)=-V(z) \neq 0$, one can define a new coordinate $t$ by means of $d t=d v-d z / V(z)$ so that the line-element in case (B.ii) with $\epsilon=1$ becomes

$$
d s^{2}=-V(d t-\hat{\boldsymbol{w}})^{2}+\frac{1}{V} d z^{2}+\left(k^{2}+z^{2}\right) h_{+} .
$$

This form corresponds to the $\Lambda$-vacuum solutions presented in formulae [(2.6) plus (4.33)] or [(2.8) plus (4.43)] of [5], the former for the case with $V<0$ having a 4-dimensional group of motions acting on timelike hypersurfaces, the latter for $V>0$ and has a 4-dimensional group of motions acting on spacelike hypersurfaces (see alternatively [(13.9) plus (13.48)] without charge in [45]). The Taub-NUT case with a cosmological constant corresponds to the case where $h_{+}$has positive constant curvature.

Finally, to prove point 4 , and as the case (B.ii) with $\epsilon=-1$ corresponds to the special case with $g(\xi, k)=g(\xi, \ell)=0$, it is easily checked from (50) and (51) that both principal null directions have zero expansion and twist. Hence, they belong to the $\Lambda$-vacuum Petrov type-D Kundt class. They actually exhaust this class, as can be seen by simply comparing the metric with that in the discussion in section 7.2.1 of [16] -alternatively, with expression (31.61) in [45] without the electromagnetic charges plus the $\Lambda$ term mentioned in p.484 of that reference. These solutions were presented also in [5] as [(2.11) plus (4.61)] and thus they possess a 4-dimensional group of motions acting on spacelike hypersurfaces everywhere.

We can finally obtain the characterization of the Kerr-NUT-(A)dS metric as defined in the Introduction.

Theorem 6. With the same hypothesis as in Theorem 4, assume that there is one point $p \in \mathcal{M}$ where $Q \mathcal{F}^{2}-4 \Lambda \neq 0$ (and hence everywhere), and that at least at one point $\xi$ is not orthogonal to the plane spanned by the two real null eigenvectors of $\mathcal{F}_{\alpha \beta}$. Let $b_{1}, b_{2}, c$ be as in Theorem 4 and define $P:=\frac{6 i \sqrt{\mathcal{F}^{2}}}{{Q \mathcal{F}^{2}-4 \Lambda}^{2}}$ and $y, Z: \mathcal{M} \rightarrow \mathbb{R}$ by $P:=y+i Z$. Then the function

$$
k=\left(y^{2}+Z^{2}\right) \nabla_{\alpha} Z \nabla^{\alpha} Z-b_{2} Z+c Z^{2}+\frac{\Lambda}{3} Z^{4}
$$

is constant on $\mathcal{M}$. If the polynomial

$$
V(\zeta):=k+b_{2} \zeta-c \zeta^{2}-\frac{\Lambda}{3} \zeta^{4}
$$

admits two zeros $\zeta_{0} \leq \zeta_{1}$ such that the factor polynomial $\hat{V} \equiv V\left(\zeta-\zeta_{0}\right)^{-1}\left(\zeta_{1}-\zeta\right)^{-1}$ is strictly positive on $\left[\zeta_{0}, \zeta_{1}\right]$ and $Z$ takes values in $\left[\zeta_{0}, \zeta_{1}\right]$ then the spacetime $(\mathcal{M}, g)$ is locally isometric to the Kerr-NUT-(A)dS with parameters $\{\Lambda, m, a, l\}$ where

$$
m=\frac{b_{1}}{2 v_{0} \sqrt{v_{0}}}, \quad a=\frac{\zeta_{1}-\zeta_{0}}{2 \sqrt{v_{0}}}, \quad l=\frac{\zeta_{1}+\zeta_{0}}{2 \sqrt{v_{0}}}
$$

and $v_{0}:=\hat{V}\left(\frac{\zeta_{0}+\zeta_{1}}{2}\right)$. 
Remark. It is immediate to check that the characterization Theorem 1 stated in the introduction is a combination of Theorems 4 and 6 ,

Proof: If $Z$ is constant on $\mathcal{M}$, the constancy of $k$ is trivial. Otherwise, its constancy has been shown in the proof of Lemma 13 with $u$ as given in Theorem 2, The conditions $V\left(\zeta_{0}\right)=V\left(\zeta_{1}\right)=0$ and the definitions of $l, a, v_{0}$ imply

$$
b_{2}=2 v_{0} \sqrt{v_{0}} l\left(1+\frac{\Lambda}{3}\left(a^{2}-4 l^{2}\right)\right), \quad k=v_{0}^{2}\left(a^{2}-l^{2}\right)\left(1-l^{2} \Lambda\right), \quad c=v_{0}\left(1-\frac{\Lambda}{3}\left(a^{2}+6 l^{2}\right)\right),
$$

and the polynomial $\hat{V}$ reads $\hat{V}=v_{0}\left(1+\frac{\Lambda}{3}\left(\frac{\zeta}{\sqrt{v_{0}}}-l\right)\left(\frac{\zeta}{\sqrt{v_{0}}}+3 l\right)\right)$. Assume first that $\zeta_{1}-\zeta_{0}>0$ (i.e. $a \neq 0$ ). We first show that there is no point where $d Z$ and $\nabla_{\mu} \nabla^{\mu} Z$ vanish simultaneously. Indeed, if this were the case, then $Z$ would be constant on $\mathcal{M}$ (see the Remark after Proposition (6), which, given the definitions (90), (91) and the hypothesis Image $(Z) \subset$ $\left[\zeta_{0}, \zeta_{1}\right]$, can only happen if $Z=\zeta_{i}(i=0,1)$ everywhere. In either case, expression (59) with $\epsilon=1$ becomes $2 a v_{0} \sqrt{v_{0}} \hat{V}\left(\zeta_{i}\right)=0$, against hypotheses. Thus, we are in case (B.i) of Theorem 4. Moreover, if $Z$ takes somewhere the values $\zeta_{0}$ or $\zeta_{1}$, it does so (by Proposition 6) on a totally geodesic 2 -dimensional timelike surface where a Killing vector vanishes. Away from these points we can perform the coordinate changes

$$
Z=\sqrt{v_{0}}(l+a \cos \theta), \quad y=\sqrt{v_{0}} r, \quad v=\frac{u}{\sqrt{v_{0}}}+v_{0}(a+l)^{2} x, \quad x=-\frac{\phi}{v_{0} \sqrt{v_{0}} a},
$$

which brings the metric of case (B.i) in Theorem 4 into (11).

If on the other hand $\zeta_{0}=\zeta_{1}$ (i.e. $a=0$ ), then $Z=\zeta_{0}=\sqrt{v_{0}} l$ is constant and we are in case (B.ii) of Theorem 4 with $\epsilon=1$. Given the relation $c=-2 \Lambda v_{0} l^{2}+\kappa$ (see the proof of Theorem (4) it follows $\kappa=v_{0}>0$ and hence $h_{+}=\left(1 / v_{0}\right) \gamma$ where $\gamma$ is the standard metric of the sphere. Since $\beta=Z=\sqrt{v_{0}} l$, equation (89) becomes $\hat{d} \hat{\boldsymbol{w}}=\left(2 l / \sqrt{v_{0}}\right) \boldsymbol{\eta}_{\boldsymbol{\gamma}}$, where $\boldsymbol{\eta}_{\boldsymbol{\gamma}}$ the volume form of $\gamma$. The general solution to this equations is, in standard spherical coordinates $\{\theta, \phi\}, \hat{\boldsymbol{w}}=\left(4 l / \sqrt{v_{0}}\right) \sin ^{2}(\theta / 2) d \phi+d f_{0}$, where $f_{0}$ is any smooth function on the sphere. With the change of variables $y=\sqrt{v_{0}} r$ and $v=f_{0}+u / \sqrt{v_{0}}$ and the redefinition $n=-2 v_{0} \sqrt{v_{0}} m$, the metric in case (B.ii) $(\epsilon=1)$ of Theorem 1 becomes (1) with $a=0$.

\subsection{Comments on Theorem 6 and remarks concerning other re- lated metrics}

As we have seen, the spacetime characterization of the Kerr-NUT-(A)dS metric given in Theorem 6 requires some extra conditions, apart from our main assumption (87). Even though those conditions may look somehow artificial, they are actually required and generalize similar previous conditions used to characterize other simpler metrics. For instance, Theorem [6 includes and extends the characterization of the Kerr metric - and of the KerrNUT metric [32] - as originally given in [30, 31] and recently complemented and corrected in [35] with the necessary, but only implicitly assumed in [30, 31], condition that $\xi$ must be 
non-orthogonal to the 2-plane spanned by the real null eigenvectors of $\mathcal{F}_{\alpha \beta}$ somewhere. Observe that this omitted assumption is actually redundant whenever $\xi$ is timelike somewhere. Taking this assumption into account we note that, in the $\Lambda=0$ case, the condition that the polynomial (91) has two zeros $\zeta_{0}, \zeta_{1}$ and the factor polynomial $\hat{V}$ is positive between them is simply equivalent to $c>0$, which is the characterization of the Kerr-NUT metric as given in Theorem 3 in [32]. The Kerr subcase corresponds to the NUT parameter $l$ being zero and $m \neq 0$, or equivalently $b_{1} \neq 0$ and $b_{2}=0$ (see (92)). Since in the case $\Lambda=0$ we have from (88):

$$
\Lambda=0 \Longrightarrow \mathcal{F}^{2} Q^{4}\left(-b_{2}+i b_{1}\right)^{2}=36^{2}
$$

the conditions $b_{1} \neq 0=b_{2}$ can be equivalently written by demanding that the constant $\mathcal{F}^{2} Q^{4}$ is real and negative, which agrees with the statement of Theorem 1 in [31].

The question arises about metrics for which the polynomial (91) does not satisfy the conditions of Theorem [6] — while still satisfying the main assumption (87). We do not intend to give an exhaustive discussion here. We just mention two known classes solutions which do satisfy our main characterization hypothesis (87) but not the conditions on the zeroes of the polynomial $V(\zeta)$ in Theorem 6. More precisely, in [27] two families of metrics with negative cosmological constant are constructed. First, the family of metrics (10) in [27] is obtained by analytic continuation of the parameters of the Kerr-de Sitter metric with vanishing NUT parameter $l=0$. Second, the family (43) in [27] is built by direct choice of parameters in the Plebanski solution. Thereby, these two classes of $\Lambda$-vacuum solutions also satisfy our main algebraic constraint (87), thus they must belong to the general family in Theorem 4. It turns out that the two classes have $b_{2}=0$, and are then distinguished as follows (keeping $\Lambda<0$ )

- The first class, (10) in [27], corresponds to the situation when furthermore the constant $k$ defined as in Theorem 6 is negative. In this case, the polynomial $V(\zeta)$ has precisely two real zeros $-\zeta_{1}=\zeta_{2}>0$ and $V(\zeta) \geq 0$ on the set $\mathcal{D}_{0}:=\left(-\infty,-\zeta_{2}\right] \cup\left[\zeta_{2}, \infty\right)$. The solution is recovered with $Z: \mathcal{M} \rightarrow \mathbb{R}$ taking values in either of the two connected components of $\mathcal{D}_{0}$. Not surprisingly, the analytic continuation of the parameters performed in 27] changes drastically the structure of the domains where the polynomial $V(\zeta)$ is positive (in this case, it transforms a compact interval into a non-compact closed interval). It is precisely this domain structure that plays a crucial role in determining the local form of the metric. This is why the statement of Theorem 6 characterizing the Kerr-NUT-(A) de Sitter metric requires fixing one such domain structure.

- The second class, (43) in [27], has $V(\zeta)>0$ everywhere, so that $V(\zeta)$ has no zeros, and moreover $c=0$ which implies also that $k>0$. Thus, $V(\zeta)$ has a unique minimum at the origin. This class of metrics, have been considered in the context of the AdS/CFT correspondence as viable models of an holographic description for the Quark-Gluon plasma [36, 37].

Actually, a generalization of the last case has also been recently analyzed in [38]. The metric (21) in [38] is simply the case (keeping $\Lambda<0, b_{2}=0$ ) with no zeros for $V(\zeta)$, which 
has $k>0$ necessarily and is defined by $c^{2}+4 k \Lambda / 3<0$, plus the condition $c>0$, ergo $V(\zeta)$ has a local maximum at the origin.

It seems therefore advisable to perform a complete analysis of the different qualitative possibilities for the function (91) according to its roots and local extrema, see [18] pp. 309 and following, where several results along these lines can be found.

\section{Acknowledgements}

The authors wish to thank Walter Simon for comments on the manuscript and for providing useful references. MM acknowledges financial support under the project FIS2012-30926 (MICINN). JMMS is supported by grants FIS2010-15492 (MICINN), GIU12/15 (Gobierno Vasco) and UFI 11/55 (UPV/EHU). The authors acknowledge support from project P09FQM-4496 (J. Andalucía-FEDER).

\section{Appendix}

In this Appendix we collect some of the formulas that are used in the main text.

$$
\begin{aligned}
& \mathcal{F}_{\mu}{ }^{\rho} t_{\nu \rho}=\frac{1}{8} \mathcal{F}^{2} \overline{\mathcal{F}}_{\mu \nu}, \\
& \xi^{\mu} t_{\mu \nu}=-\frac{1}{4} \eta_{\nu}, \quad \chi^{\mu} t_{\mu \nu}=\frac{1}{8} \mathcal{F}^{2} \bar{\chi}_{\nu}, \quad \eta^{\mu} t_{\mu \nu}=-\frac{1}{16} \mathcal{F}^{2} \overline{\mathcal{F}}^{2} \xi_{\nu}, \\
& \xi^{\mu} \xi^{\nu} t_{\mu \nu}=\frac{1}{8} \chi_{\rho} \bar{\chi}^{\rho}, \quad \xi^{\mu} \chi^{\nu} t_{\mu \nu}=0, \quad \xi^{\mu} \eta^{\nu} t_{\mu \nu}=\frac{N}{16} \mathcal{F}^{2} \overline{\mathcal{F}}^{2}, \quad \chi^{\mu} \eta^{\nu} t_{\mu \nu}=0, \\
& \chi^{\mu} \chi^{\nu} t_{\mu \nu}=\frac{1}{8} \mathcal{F}^{2} \chi_{\rho} \bar{\chi}^{\rho}, \quad \chi^{\mu} \bar{\chi}^{\nu} t_{\mu \nu}=-\frac{N}{8} \mathcal{F}^{2} \overline{\mathcal{F}}^{2}, \quad \eta^{\mu} \eta^{\nu} t_{\mu \nu}=\frac{1}{32} \mathcal{F}^{2} \overline{\mathcal{F}}^{2} \chi_{\rho} \bar{\chi}^{\rho} .
\end{aligned}
$$

For arbitrary (complex or real) 2-forms $A_{\mu \nu}$ and $B_{\mu \nu}$, the Lanczos identity for double 2-forms - expression (A15) in p.2839 of [42] - applied to $A_{\alpha \beta} B_{\mu \nu}$ implies the 4-dimensional identity

$$
A_{\alpha \beta} B_{\mu \nu}+B_{\alpha \beta}^{\star} A_{\mu \nu}^{\star}=L_{\mu \alpha} g_{\beta \nu}-L_{\nu \alpha} g_{\beta \mu}-L_{\mu \beta} g_{\alpha \nu}+L_{\nu \beta} g_{\alpha \mu}
$$

where $L_{\mu \nu} \stackrel{\text { def }}{=} B_{\mu}{ }^{\rho} A_{\nu \rho}-(1 / 4) g_{\mu \nu} B^{\rho \sigma} A_{\rho \sigma}$. In particular, one has the identity

$$
\mathcal{F}_{\alpha \beta} \overline{\mathcal{F}}_{\mu \nu}+\overline{\mathcal{F}}_{\alpha \beta} \mathcal{F}_{\mu \nu}=2\left(t_{\alpha \mu} g_{\beta \nu}-t_{\alpha \nu} g_{\beta \mu}-t_{\beta \mu} g_{\alpha \nu}+t_{\beta \nu} g_{\alpha \mu}\right)
$$


Different contractions here lead to

$$
\begin{aligned}
& 4 t_{\beta[\mu} \xi_{\nu]}=g_{\beta[\mu} \eta_{\nu]}-\frac{1}{2} \chi_{\beta} \overline{\mathcal{F}}_{\mu \nu}-\frac{1}{2} \bar{\chi}_{\beta} \mathcal{F}_{\mu \nu}, \\
& 2 N t_{\beta \mu}=\frac{1}{4} \chi_{\rho} \bar{\chi}^{\rho} g_{\beta \mu}+\xi_{(\beta} \eta_{\mu)}+\frac{1}{8} \chi_{(\beta} \bar{\chi}_{\mu)}, \\
& 4 t_{\beta[\mu} \chi_{\nu]}=\frac{1}{2} \mathcal{F}^{2}\left(\xi_{\beta} \overline{\mathcal{F}}_{\mu \nu}-g_{\beta[\mu} \bar{\chi}_{\nu]}\right)-\eta_{\beta} \mathcal{F}_{\mu \nu}, \\
& 2\left(\chi_{\rho} \bar{\chi}^{\rho}\right) t_{\beta \mu}=\eta_{\beta} \eta_{\mu}+\frac{1}{4}\left(\overline{\mathcal{F}}^{2} \chi_{\beta} \chi_{\mu}+\mathcal{F}^{2} \bar{\chi}_{\beta} \bar{\chi}_{\mu}\right)+\frac{1}{4} \mathcal{F}^{2} \overline{\mathcal{F}}^{2}\left(\xi_{\beta} \xi_{\mu}+N g_{\beta \mu}\right) \\
& 4 t_{\beta[\mu} \eta_{\nu]}=\frac{1}{4} \mathcal{F}^{2} \overline{\mathcal{F}}^{2} g_{\beta[\mu} \xi_{\nu]}+\frac{1}{4} \overline{\mathcal{F}}^{2} \chi_{\beta} \mathcal{F}_{\mu \nu}+\frac{1}{4} \mathcal{F}^{2} \bar{\chi}_{\beta} \overline{\mathcal{F}}_{\mu \nu}
\end{aligned}
$$

Contracting (98, 100, 102) with $k_{ \pm}$one gets

$$
\begin{aligned}
& -2 R \bar{R} \boldsymbol{k}_{ \pm} \wedge \boldsymbol{\xi}=\boldsymbol{k}_{ \pm} \wedge \boldsymbol{\eta} \mp 2 g\left(\xi, k_{ \pm}\right)(R \overline{\mathcal{F}}+\bar{R} \mathcal{F}) \\
& -R \bar{R} \boldsymbol{k}_{ \pm} \wedge \chi=R^{2} \boldsymbol{k}_{ \pm} \wedge \bar{\chi}-2 R g\left(\xi, k_{ \pm}\right)(R \overline{\mathcal{F}}+\bar{R} \mathcal{F}) \\
& -R \bar{R} \boldsymbol{k}_{ \pm} \wedge \boldsymbol{\eta}=2(R \bar{R})^{2} \boldsymbol{k}_{ \pm} \wedge \boldsymbol{\xi} \mp 2 R \bar{R} g\left(\xi, k_{ \pm}\right)(R \overline{\mathcal{F}}+\bar{R} \mathcal{F})
\end{aligned}
$$

Observe that (105) is simply (103) multiplied by $R \bar{R}$.

Using again the identity (97) we derive

$$
4 t_{\beta[\mu} k_{\nu]}^{ \pm}=2 R \bar{R} g_{\beta[\mu} k_{\nu]}^{ \pm} \pm k_{\beta}^{ \pm}\left(R \overline{\mathcal{F}}_{\mu \nu}+\bar{R} \mathcal{F}_{\mu \nu}\right)
$$

and contracting here the +-equation with $k_{-}^{\beta}$

$$
R \overline{\mathcal{F}}_{\mu \nu}+\bar{R} \mathcal{F}_{\mu \nu}=-2 R \bar{R}\left(k_{\mu}^{+} k_{\nu}^{-}-k_{\mu}^{-} k_{\nu}^{+}\right)
$$

or with $k_{-}^{\mu}$

$$
-2 g\left(k_{+}, k_{-}\right) t_{\mu \nu}=R \bar{R}\left[2 k_{\mu}^{+} k_{\nu}^{-}+2 k_{\mu}^{-} k_{\nu}^{+}-g\left(k_{+}, k_{-}\right) g_{\mu \nu}\right]
$$

and both equations with $\xi$

$$
2 g\left(k_{ \pm}, \xi\right) t_{\mu \nu}=R \bar{R} g\left(k_{ \pm}, \xi\right) g_{\mu \nu}-\frac{1}{2} k_{\nu}^{ \pm}\left(\eta_{\mu}+2 R \bar{R} \xi_{\mu}\right) \mp \frac{1}{2} k_{\mu}^{ \pm}\left(R \bar{\chi}_{\nu}+\bar{R} \chi_{\nu}\right) .
$$

Equations (103) and (104) - or directly (109) - imply necessarily

$$
\boldsymbol{k}_{ \pm} \wedge(\boldsymbol{\eta}+2 R \bar{R} \boldsymbol{\xi} \mp R \overline{\boldsymbol{\chi}} \mp \bar{R} \boldsymbol{\chi})=0
$$

whose general solution reads

$$
\boldsymbol{\eta}+2 R \bar{R} \boldsymbol{\xi} \mp R \overline{\boldsymbol{\chi}} \mp \bar{R} \boldsymbol{\chi}=A_{ \pm} \boldsymbol{k}_{ \pm}
$$

for real $A_{ \pm}$with

$$
g\left(k_{+}, k_{-}\right) A_{ \pm}=8 R \bar{R} g\left(k_{\mp}, \xi\right), \quad A_{ \pm} g\left(k_{ \pm}, \xi\right)=-2 N R \bar{R}-\frac{1}{2} g(\chi, \bar{\chi}) .
$$

The above is valid for both the regular and singular cases. In this paper we are only concerned with the regular case, and thus $\mathcal{F}^{2}=-4 R^{2} \neq 0$. We can always normalize the null eigenvectors such that $g\left(k_{+}, k_{-}\right)=-1$ so that (108) becomes

$$
2 t_{\mu \nu}=R \bar{R}\left[g_{\mu \nu}+2 k_{\mu}^{+} k_{\nu}^{-}+2 k_{\mu}^{-} k_{\nu}^{+}\right] \text {. }
$$




\section{References}

[1] S. Alexakis, A.D. Ionescu, S. Klainerman, "Uniqueness of smooth stationary black holes in vacuum: small perturbations of the Kerr spaces" (2010) Commun. Math. Phys. 299 89-127.

[2] S. Alexakis, A.D. Ionescu, S. Klainerman, "Hawking's local rigidity theorem without analyticity" (2010) Geom. Funct. Anal. 20 845-869.

[3] S. Alexakis, A. D. Ionescu, S. Klainerman, "Rigidity of stationary black holes with small angular momentum on the horizon" (2013) arXiv:1304.0487 [gr-qc]

[4] T. Bäckdahl, J.A. Valiente Kroon, "On the construction of a geometric invariant measuring the deviation from Kerr data" (2010) Ann. Henri Poincaré 11 1225-1271.

[5] M. Cahen, L. Defrise, "Lorentzian 4 dimensional manifolds with 'local isotropy" , (1968) Commun. Math. Phys. 11 56-76.

[6] W. Chen, H. Lü, C.N. Pope, "General Kerr-NUT-AdS metrics in all dimensions" (2006) Class. Quant. Grav. 23 5323-5340.

[7] Z-W. Chong, G.W. Gibbons, H. Lü. C.N. Pope, "Separability and Killing Tensors in Kerr-Taub-Nut-De Sitter Metrics in Higher Dimensions" (2005) Phys. Lett. B 609 124-132.

[8] M. Dafermos, I. Rodnianski, "Lectures on black holes and linear waves", in Evolution equations, Clay Mathematics Proceedings, Vol. 17. Amer. Math. Soc., Providence, RI, (2013) 97-205 (online at http://www.arxiv.org/abs/0811.0354).

[9] R. Debever, N. Kamran, R.G. McLenaghan, "Exhaustive integration and a single expression for the general solution of the type $\mathrm{D}$ vacuum and electrovac field equations with cosmological constant for a nonsingular aligned Maxwell field" (1984) J. Math. Phys. 25 1955-1972.

[10] O.J.C. Dias, G.T. Horowitz, D. Marolf, J.E. Santos, "On the nonlinear stability of asymptotically anti-de Sitter solutions", (2012) Class. Quant. Grav. 29230519.

[11] S. Dyatlov, "Exponential energy decay for Kerr-de Sitter black holes beyond event horizons" (2011) Math. Res. Lett. 18 1023-1035.

[12] J. J. Ferrando, J. A. Sáez, "On the invariant symmetries of the D-metrics", (2007) J. Math. Phys. 48102504.

[13] A. García-Díaz, "Electrovac type D solutions with cosmological constant" (1984) J. Math. Phys. 25 1951-1954.

[14] A. García-Parrado Gómez-Lobo, J.M.M. Senovilla, "A set of invariant quality factors measuring the deviation from the Kerr metric" (2013) Gen. Rel. Grav. 45 1095-1127 
[15] A. García-Parrado Gómez-Lobo, J.A. Valiente Kroon "Kerr Initial data" (2008) Class. Quant. Grav. 25205018.

[16] J.B. Griffiths, J. Podolský, "A new look at the Pelbański-Demiański family of solutions", (2006) Int. J. Mod. Phys. D 15 335-370.

[17] J.B. Griffiths, J. Podolský, "A note on the parameters of the Kerr-NUT-(anti-)de Sitter spacetime", (2007) Class. Quant. Grav. 24 1687-1689.

[18] J.B. Griffiths, J. Podolský, Exact Space-Times in Eintein's General Relativity, Cambridge Monographs on Mathematical Physics (Cambridge Univ. Press, Cambridge, 2009)

[19] D. Gromoll, G. Walschap, "Metric foliations and curvature", Progress in Mathematics 268 (Birkhäuser Verlag AG, Basel, 2009).

[20] G. Holzegel, "On the massive wave equation on slowly rotating Kerr-AdS spacetimes", (2010) Commun. Math. Phys. 294 169-197.

[21] G. Holzegel, J. Smulevici, "Decay Properties of Klein-Gordon Fields on Kerr-AdS spacetimes", (2011) arXiv:1110.6794 [gr-qc].

[22] T. Houri, T. Oota, Y. Yasui, "Closed conformal Killing-Yano tensor and Kerr-NUTde Sitter space-time uniqueness" (2007) Phys. Lett. B 656 214-216.

[23] T. Houri, T. Oota, Y. Yasui, "Closed conformal Killing-Yano tensor and the uniqueness of generalized Kerr-NUT-de Sitter spacetime", (2009) Clas. Quant. Grav. 26 045015 .

[24] A.D. Ionescu, S. Klainerman, "On the uniqueness of smooth, stationary black holes in vacuum", (2009) Invent. Mathem. 175 35-102.

[25] A.D. Ionescu, S. Klainerman, "On the local extension of Killing vector-fields in Ricci flat manifolds", (2013) J. Amer. Math. Soc. 26 563-593.

[26] W. Israel, "Differential forms in general relativity", (1970) Commun. of the Dublin Institute for Advanced Studies, Series A, 19 1-100.

[27] D. Klemm, V. Moretti, L. Vanzo, "Rotating topological black holes", (1998) Phys. Rev. D 57 6127-6137. Erratum, (1999) ibid. 60109902.

[28] P. Krtous, V.P. Frolov, D. Kubiznak, "Hidden symmetries of higher-dimensional black holes and uniqueness of the Kerr-NUT-(A)dS spacetime", (2008) Phys. Rev. D 78064022 .

[29] D. Kubiznak, V.P. Frolov, "The hidden symmetry of higher dimensional Kerr-NUTAdS spacetimes" (2007) Class. Quant. Grav. 24 F1-F6. 
[30] M. Mars, "A spacetime characterization of the Kerr metric", (1999) Class. Quantum. Grav. 16 2507-2523.

[31] M. Mars, "Uniqueness properties of the Kerr metric", (2000) Class. Quantum Grav. $173353-3373$.

[32] M. Mars, "Spacetime Ehlers group: transformation law for the Weyl tensor", (2001) Clas. Quant. Grav. 18 719-738.

[33] M. Mars, "Wahlquist-Newman solution", (2001) Phys. Rev. D 63064022.

[34] M. Mars, M. Reiris, "Global and uniqueness properties of stationary and static spacetimes with outer trapped surfaces", Commun. Math. Phys. (2013), (DOI) $10.1007 / \mathrm{s} 00220-013-1739-5$.

[35] M. Mars and J.M.M. Senovilla, "Spacetime characterizations of $\Lambda$-vacuum metrics with a null Killing 2-form" (2016) Class. Quantum Grav. 33195004

[36] B. McInnes, "Fragile black holes and an angular momentum cutoff in peripheral heavy ion collision" (2012) Nucl. Phys. B 861236.

[37] B. McInnes, "Universality of the holographic angular momentum cutoff" (2012) Nucl. Phys. B 864722.

[38] B. McInnes and E. Teo, "Generalised planar black holes and the holography of hydrodynamic shear" (2013) arXiv:1309.2054.

[39] B. O'Neill, "The fundamental equations of a submersion", (1966) Michigan Math. J. 13 459-469.

[40] A. Papapetrou, "Champs gravitationnels stationnaires à symétrie axiale", (1966) Annales de l'institut Henri Poincaré (A) Physique théorique 4, 83-105.

[41] Z. Perjés, "An improved characterization of the Kerr metric", (1985) KFKI-1984-115 preprint, in Quantum Gravity 3, Ed. M.A. Markov, World Scientific Publishing Co., Singapore.

[42] J.M.M. Senovilla, "Super-energy tensors", (2000) Class. Quantum Grav. 17, 27992841.

[43] W. Simon, "Characterizations of the Kerr metric", (1984) Gen. Rel.Grav. 16 465476.

[44] W. Simon, "Nuts have no hair", (1995) Class. Quantum Grav. 12 L125-L130.

[45] H. Stephani, D. Kramer, M. MacCallum, C. Hoenselaers, E. Herlt, Exact solutions of Einstein's field equations, 2nd ed., Cambridge Monographs on Mathematical Physics (Cambridge University Press, Cambridge, 2003). 
[46] W.W-Y. Wong, "A space-time characterization of the Kerr-Newman metric", (2009) Ann. Henri Poincaré 10 453-484. 